\title{
Petrogenesis of granitoid rocks at the northern margin of the Eastern Ghats Mobile Belt and evidence of syn-collisional magmatism
}

\author{
S Bhattacharya ${ }^{1 *}, \mathrm{RAJib}_{\mathrm{KaR}}{ }^{2}$ and S Moitra ${ }^{1}$ \\ ${ }^{1}$ Indian Statistical Institute, 203 B.T. Road, Kolkata 700 108, India. \\ ${ }^{2}$ Department of Geology, J.K. College, Purulia 723 101, India. \\ *e-mail:samar@isical.ac.in
}

The northern margin of the Eastern Ghats Mobile belt against the Singhbhum craton exposes granitic rocks with enclaves from both the high-grade and low-grade belts. A shear cleavage developed in the boundary region is also observed in these granitoids. Field features and petrography indicate syn-tectonic emplacement of these granitoids. Petrology-mineralogy and geochemistry indicate that some of the granitoids are derived from the high-grade protoliths by dehydration melting. Others could have been derived from low-grade protoliths. Moreover, microstructural signatures in these granitoids attest to their syn-collisional emplacement.

\section{Introduction}

The Eastern Ghats Mobile Belt (EGMB) along the east coast of India is bounded by the granitegreenstone terrain of the Singhbhum craton (SC) to the north. Several workers have reported the occurrence of granitic rocks in the boundary region (Banerjee et al 1987; Patra et al 1994; Mahalik 1994). On the evidence of blastomylonites in the boundary region, Banerjee et al (1987) proposed a faulted boundary and from Landsat imagery Mahalik (1994) proposed a boundary fault. From a detailed analysis of structures in the two adjoining belts, namely EGMB and SC, around Bhuban, Bhattacharya (1997) proposed an oblique collision, whereby the EGMB collided with the stable SC. Isoclinal and rootless $F_{1}$ folds with NE-SW trending steep axial plane foliation $S_{1}$ suggest a regional NW-SE directed compression during the development of the first generation folds in the EGMB. The tectonic juxtaposition by oblique collision (transpression), is indicated by a south to north convergent movement in the granulite belt of the Eastern Ghats and strike-slip shear zones in the rocks of the boundary region (Bhattacharya 1997). Moreover, on the evidence of granulitic rocks recorded from the boreholes in Rengali Dam-site (where exposed lithologies are quartzites of the adjoining Singhbhum craton), Bhattacharya (1997) argued that Eastern Ghats rocks moved below the supracrustals of the SC by a northward convergent motion. Although the age relationship between the supracrustals of the Singhbhum craton and that of the Eastern Ghats Mobile belt has not yet been established with isotopic data, some isotopic data from the granulites of the adjoining Eastern Ghats (Bhattacharya et al 2001) and granitoids of the boundary region (Misra et al 2000) suggest granitic magmatism (ca. $2.8 \mathrm{Ga}$ ) in the marginal parts of the Eastern Ghats belt closely followed granulite facies event (ca. 3.0 Ga) in the adjoining belt of the Eastern Ghats.

However, no in-depth study of the petrologygeochemistry of the granitoids of the boundary region and their relation to tectonism has yet been undertaken. In this communication, we present petrological and geochemical data on some granitoid rocks of the boundary region around Bhuban.

Keywords. Eastern Ghats; Singhbhum craton; enclaves; different protoliths; collisional magmatism. 


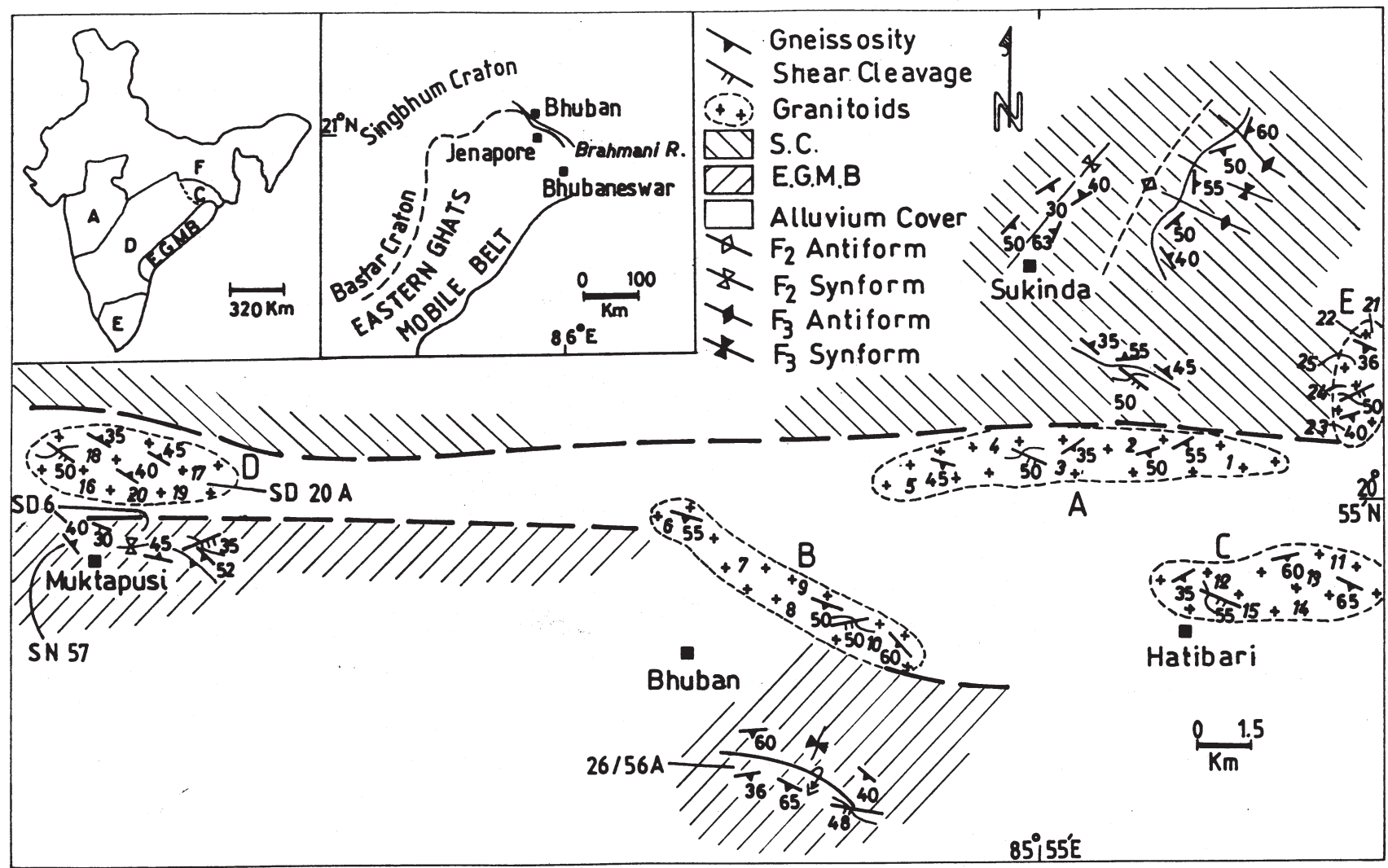

Figure 1. Simplified geological map, showing the disposition of the granitoids in relation to the two crustal provinces of the Eastern Ghats mobile belt and the Singhbhum craton. Note pervasive foliations in the two provinces are discordant to each other and hence folds in the two provinces developed independently. Locations of analyzed samples are also shown.

In addition, we present several key features on mesoscopic to microscopic scales, which attest to the relation of the granitic magmatism with collisional tectonism.

\section{Geological setting}

The study area around Bhuban in Orissa (figure 1, inset) is located on the northern margin of the Eastern Ghats mobile belt against the Singhbhum craton. A host of granitoids separates the two lithotectonic provinces (figure 1).

\subsection{Eastern ghats}

In the study area lithologies include charnockiteenderbite gneisses, mafic granulites, garnetsillimanite-K-feldspar gneisses (khondalite) and garnet-bearing quartzites (Moitra 1996). Moitra (1996) also recorded three phases of folding and corresponding foliation development in the granulites. Bhattacharya (1997) described a post- $F_{3}$ shear cleavage and associated blastomylonites. Interestingly, this feature is restricted to the boundary region and ubiquitous in both the lithotectonic provinces.

\subsection{Singhbhum craton}

Lithologies include quartzites, cherty quartzites and banded hematite quartzites and intrusive dolerite dykes. Moitra (1996) described three phases of folding and associated foliation development also in this province; near the boundary a post- $F_{3}$ shear cleavage is observed and cuts the $S_{3}$ cleavage.

It is important to note that folding in the two adjoining crustal provinces developed independently of each other; this is further indicated by the discordant relation between the pervasive foliations in the two provinces: ESE-WNW in the Eastern Ghats and NNE-SSW in the Singhbhum craton (figure 1).

\subsection{Granitoids}

A narrow zone, 2 to $5 \mathrm{~km}$ in width, separates the EGMB from SC and several granitoid rocks are exposed in this zone. It is important to note that EGMB and SC rocks are never in direct contact 


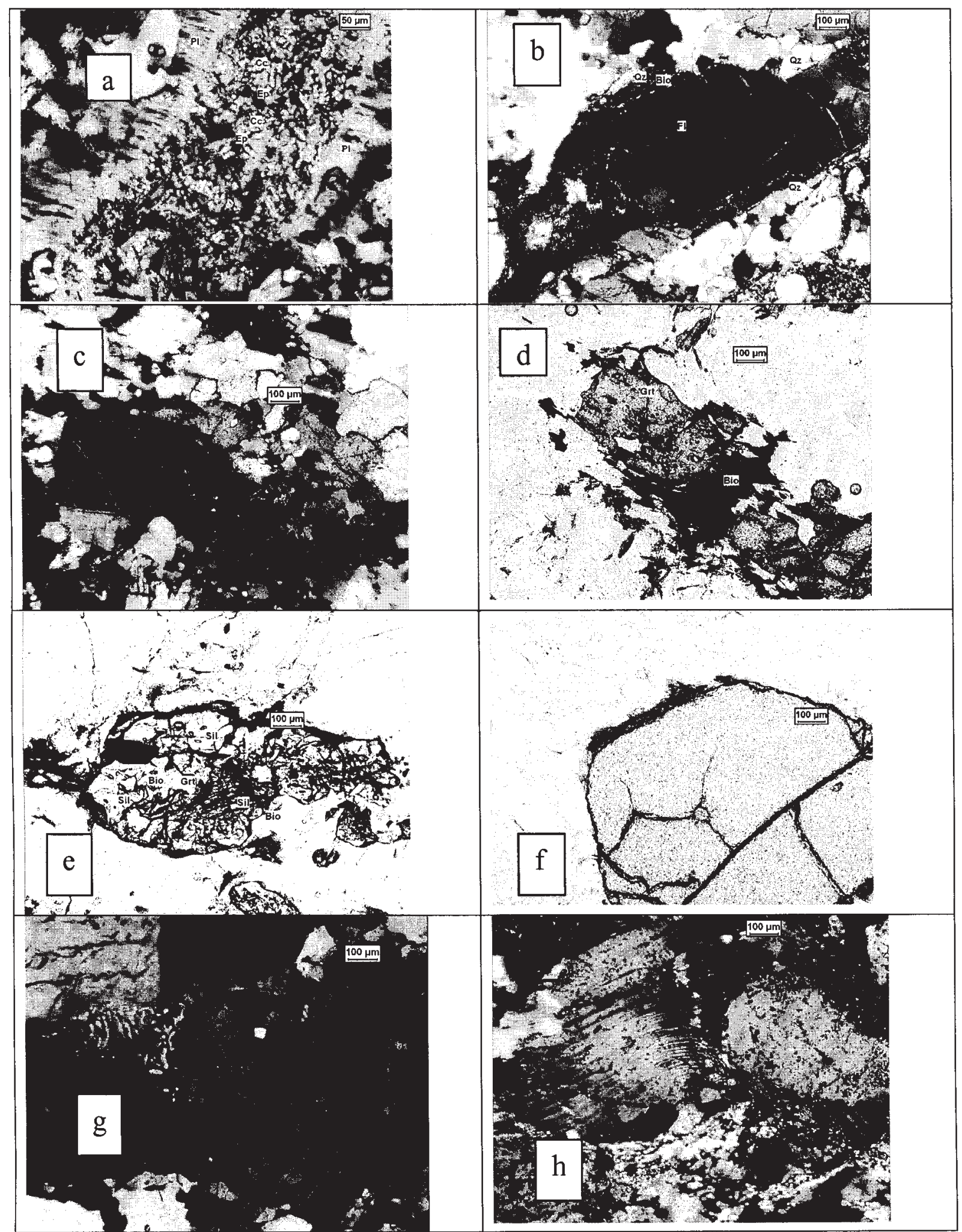

Figure 2. Photomicrographs. (a) Porphyroclastic plagioclase grains are altered to epidote plus calcite aggregates. (b) Biotite + quartz symplectite describe an anastomosing fabric around feldspar porphyroclasts, and this represents the shear cleavage. (c) Sub-grain formation in microcline is a common feature and may be ascribed to syntectonic crystallization. (d) Secondary biotite on garnet, indicating retrogression on cooling. (e) Minute granules of biotite and sillimanite inclusions in garnet suggest presence of biotite and sillmanite in the source rocks. (f) Euhedral garnet in Granite D could be a peritectic product of biotite-dehydration melting. (g) Deformation-induced replacement of K-feldspar by myrmekite. (h) Bent twin lamellae and marginal granulation in plagioclase porphyroclasts. 
but both the compositional bands/gneissic foliation and the shear foliation in the granitoids are subparallel to the pervasive foliation in the host rocks.

Most of the granitoid rocks display a gneissic foliation, which is often affected by a shear cleavage (figure 4b in Bhattacharya 1997; figure 1). Another important feature of the granitoids is the presence of enclaves from both the high-grade and low-grade belts or lithotectonic provinces (cf. figure 3 in Misra et al 2000).

\section{Field relations and petrography}

Granitoid rocks in the study area occur as separate stock-like bodies, which are also characterised by different accessory ferromagnesian minerals; these are described here as five different bodies A to $\mathrm{E}$ (figure 1). Interestingly, the enclaves in the respective granitoids are also of different types and some of the enclaves and their mineralogy provide important clues to the petrogenesis of the respective granitoids.

\subsection{Granite A}

It is relatively fine-grained, has a dark-grey appearance and a fine foliation. Under the microscope a porphyroclastic texture is observed: porphyroclasts of feldspar and amphibole are set in a fine-grained quartzofeldspathic matrix and display anastomosing gneissic foliation. Porphyroclastic plagioclase grains are commonly altered to epidote plus calcite aggregates (figure 2a). The characteristic ferromagnesian phase is amphibole and the common accessory phases are opaque oxides, epidote and zircon (table 1 ). In the modal Q-A-P classification scheme of Streckeisen (1976), Granite A would be termed alkali-feldspar granite (figure 3).
Small (up to a few cm) enclaves of amphibolite are common.

\subsection{Granite B}

A gneissic banding is characteristic and is defined by segregation of biotite and opaques. Under the microscope a porphyroclastic texture is observed. Biotite and biotite + quartz symplectite commonly define an anastomosing fabric around feldspar porphyroclasts, and this represents the shear cleavage (figure $2 \mathrm{~b}$ ). Interestingly, sub-grain formation in microcline is a common feature and could be attributed to syn-tectonic recrystallisation (figure 2c). Biotite is the common ferromagnesian silicate phase and accessory phases include opaque oxides, traces of apatite and zircon (table 1). In the Q-A-P scheme, this rock type falls in the granite field (figure 3). Enclaves of different sizes (a few $\mathrm{cm}$ to about a metre) occur as discordant blocks and folded bands and commonly consist of mafic granulite.

\subsection{Granite $C$}

A crude gneissic foliation is common and leucocratic layers are occasionally observed. Under the microscope a platy granular texture, locally with porphyroclastic feldspars, can be seen. Syntectonic recrystallization is common and is evidenced by sub-grain formation and marginal granulation of feldspar porphyroblasts. Amphibole and occasional biotite are the major ferromagnesian silicate phases, and the common accessory phases are titanite and opaque oxides (table 1). Some samples contain a little andradite-rich garnet, which is also found in the amphibolite enclaves in this granite. In QA-P scheme, this rock falls in the granite field (figure 3).

Table 1. Modal data of granitoids.

\begin{tabular}{|c|c|c|c|c|c|c|c|c|c|c|c|c|}
\hline \multicolumn{6}{|c|}{ Amphibole granite A } & \multicolumn{5}{|c|}{ Biotite granite B } & \multicolumn{2}{|c|}{ Amphibole-sphene } \\
\hline Sample & $29 \mathrm{~B}$ & $41 / 8$ & $\begin{array}{c}\text { SN } \\
115 \text { B }\end{array}$ & $\begin{array}{l}\text { SN } \\
100\end{array}$ & $\begin{array}{l}\text { SN } \\
32\end{array}$ & $48 / 11$ & $\begin{array}{c}\text { SD } \\
53\end{array}$ & $\begin{array}{c}\text { SN } \\
11\end{array}$ & $77 / 30$ & $30 / 6$ & $\begin{array}{c}\mathrm{SD} \\
53 \mathrm{~A}\end{array}$ & $\begin{array}{c}\mathrm{SD} \\
79 \mathrm{~A}\end{array}$ \\
\hline Quartz & 39 & 33 & 29 & 32 & 30 & 46 & 48 & 40 & 45 & 45 & 45 & 45 \\
\hline Alk-feldspar & 30 & 31 & 35 & 31 & 20 & 27 & 23 & 36 & 38 & 38 & 30 & 30 \\
\hline Plagioclase & 15 & 23 & 14 & 10 & 20 & 12 & 17 & 10 & 14 & 13 & 15 & 10 \\
\hline Amphibole & 7 & 8 & 12 & 11 & 11 & & & & 1 & & 4 & 5 \\
\hline Biotite & 1 & & 0.6 & 1 & 1 & 11 & 10 & 11 & 6 & 6 & 1 & \\
\hline Garnet & & & & & & & & & & & 0.8 & \\
\hline Chlorite & & & & & & & & & & & & \\
\hline Epidote & trace & trace & trace & trace & trace & & & & & & & \\
\hline Opaque & 3 & 5 & 7 & 3 & 3 & 1 & 0.6 & 0.5 & 1 & 2 & 1 & 2 \\
\hline Sphene & & trace & & & & & & & & & 3 & 3 \\
\hline Zircon & trace & trace & & trace & & trace & trace & trace & trace & trace & trace & trace \\
\hline Apatite & & & & & & trace & trace & trace & trace & trace & trace & \\
\hline
\end{tabular}




\subsection{Granite D}

This is relatively coarse-grained with a gneissic foliation. Locally coarse-grained leucocratic, pink feldspar crystals define layers oriented parallel to the gneissic banding. The common ferromagnesian silicates are garnet and biotite; however, biotite is mostly secondary after garnet (figure $2 \mathrm{~d}$ ). Presence of minute granules of biotite and sillimanite inclusions in garnet suggest the presence of biotite and sillmanite in the source rocks (figure 2e). On the other hand, euhedral garnet in this granite, could be a peritectic product of melting (figure 2f). A characteristic feature is replacement of K-feldspar by myrmekite (figure $2 \mathrm{~g}$ ), which could be related to syn-tectonic recrystallisation (Simpson and Wintsch 1989). In Q-A-P scheme, granite D falls in the granite field (figure 3). Small (up to $10 \times 10 \mathrm{~cm}$ ) discordant blocks of pelitic granulite are the common enclaves.

\subsection{Granite E}

This is relatively coarse-grained, and no foliation could be detected on the mesoscopic scale. Under the microscope xenoblastic texture is observed locally, while syn-tectonic recrystallization is indicated by bent twin lamellae and marginal granulation in plagioclase porphyroclasts (figure $2 \mathrm{~h}$ ). Chlorite and epidote are the major ferromagnesian phases; amphibole and biotite are occasionally found. Trace quantities of accessory phases include opaque oxides, titanite, zircon and apatite (table 1). In Q-A-P scheme, granite $\mathrm{E}$ falls in the granite field (figure 3). Enclaves are commonly schistose and contain chlorite and tremolite-like amphibole.

On the mesoscopic scale the alternate quartzfeldspar and hornblende-biotit e layers, and the hornblende-biotite defined mineral foliation in the granitoids are subparallel and given as gneissic foliation, $S$ in figure 1 . On microscopic scale the gran- itoids commonly show development of solid-state deformation fabrics (Paterson et al 1989). Ribbons of recrystallized quartz showing no effects of strain, can be interpreted as solid-state deformation under submagmatic conditions (figure 4a). Hornblendedefined mineral foliation, $S$, is parallel to recrystallized quartz ribbons, defining a mylonitic foliation, can be interpreted as synkinematic development of magmatic and mylonitic foliation (figure 4b). Aligned quartz-feldspar porphyroclasts, defining a mylonitic foliation (a gneissic fabric on the mesoscopic scale), and development of an incipient oblique foliation, $C$ defined by mica-fish, can be interpreted as solid-state deformation at relatively high temperature conditions (figure 4c).

\section{Mineral chemistry}

Minerals were analyzed on a Jeol Jxa-8600 M microprobe at the USIC, University of Roorkee, India. $15-\mathrm{kV}$ accelerating voltage, $2 \times 10^{-8} \mathrm{amp}$ sample current and $2-\mu \mathrm{m}$ beam diameter were used.

Apart from chemical characterization of the solid solutions, the analytical data serve two purposes. First, minerals in the different granitoids and their respective enclaves could constrain the metamorphic and melting reactions. Second, minerals of the granulites in the adjoining Eastern Ghats belt could provide the basis of geothermobarometric estimates.

\subsection{Granite A (table 2)}

Amphiboles are ferrohornblende and marginal Mgenrichment $\left(X_{\mathrm{Mg}}=0.11_{\text {core }}\right.$ to $\left.0.13_{\text {rim }}\right)$, is indicative of its prograde nature. Besides the common alkali feldspars (not analyzed), plagioclase is highly sodic in composition and commonly altered to epidote plus calcite aggregates.

Amphibole in an amphibolite enclave is also ferrohornblende but is more magnesian $\left(\mathrm{X}_{\mathrm{Mg}}=0.19\right)$

Table 1. (Continued)

\begin{tabular}{|c|c|c|c|c|c|c|c|c|c|c|c|c|}
\hline \multicolumn{3}{|c|}{ Granite C } & \multicolumn{5}{|c|}{ Biotite-garnet granite D } & \multicolumn{5}{|c|}{ Chlorite-epidote granite E } \\
\hline $37 / 7$ & $39 / 7$ & $40 / 7$ & $89 / 17$ & $94 / 18$ & $19 / 6$ & $19 / 10$ & $20 / 20$ & $63 / 13$ & $62 / 13$ & $86 / 16$ & $61 / 13$ & $34 \mathrm{~B}$ \\
\hline 44 & 39 & 44 & 33 & 47 & 48 & 38 & 44 & 28 & 33 & 29 & 29 & 32 \\
\hline 39 & 49 & 33 & 50 & 39 & 42 & 40 & 39 & 35 & 29 & 48 & 48 & 46 \\
\hline 13 & 5 & 11 & 15 & 5 & 6 & 12 & 12 & 17 & 18 & 11 & 11 & 13 \\
\hline 0.4 & 3 & 6 & & & & & & 2 & 1 & 1 & & \\
\hline \multirow[t]{4}{*}{1} & 0 & 0 & 3 & 3.1 & 1 & 2 & 1 & 6 & 10 & & 1 & \\
\hline & 0.8 & 1.5 & 2 & 6 & 4 & 3 & 4 & 0 & & & & \\
\hline & & & & & & & & 3 & 3 & 7 & 7 & 5 \\
\hline & & & & & & & & 6 & 6 & 5 & 4 & 4 \\
\hline 1 & 2 & 2 & 0.5 & 0.5 & 0.5 & 0.2 & trace & 0.2 & trace & trace & trace & trace \\
\hline 3 & 3 & 3 & & & & & & trace & & trace & trace & trace \\
\hline trace & trace & & trace & trace & trace & trace & trace & trace & trace & trace & trace & trace \\
\hline trace & & trace & & & trace & & & trace & trace & trace & trace & trace \\
\hline
\end{tabular}




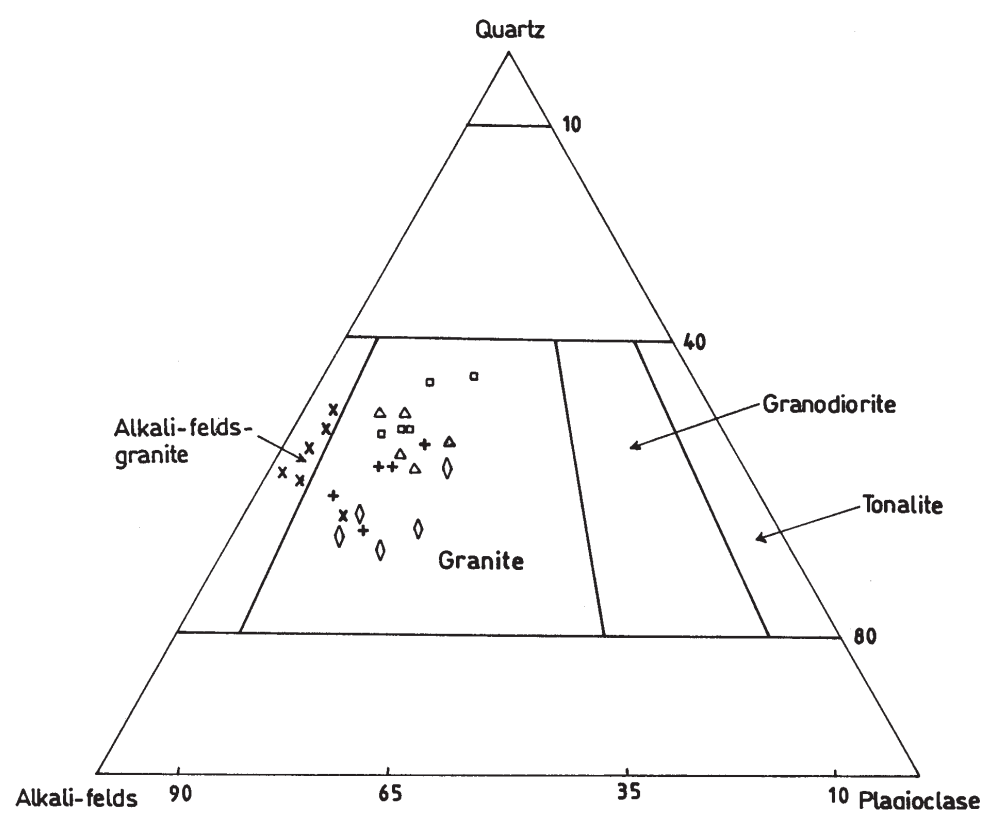

Figure 3. QAP diagram showing the modal composition of the granitoids. Symbols: Cross $=\mathbf{A}$, Box $=\mathbf{B}$, Triangle $=\mathbf{C}$, Plus $=\mathbf{D}$, Diamond $=\mathbf{E}$.

than the amphibole of the granite. Feldspar which has characteristic lamellar twinning, is pure albite $\left(X_{\mathrm{Na}}=0.98\right.$ to 0.99$)$. Because the feldspar is unaltered, its albitic composition could imply a sodic bulk composition of the precursors. The characteristic opaque oxide is ilmenite, which indicates a low oxidation state.

\subsection{Granite B (table 3)}

The biotite with marginal Mg-enrichment $\left(X_{\mathrm{Mg}}=\right.$ $0.49_{\text {core }}$ to $0.51_{\text {rim }}$ ) could be primary; however, recrystallization on shear cleavage (figure $2 \mathrm{~b}$ ) in biotite indicates its secondary nature. Plagioclase is andesine and alkali feldspar is perthite with potash feldspar host $\left(X_{\mathrm{K}}=0.9\right)$ and sodic plagioclase as blebs $\left(X_{\mathrm{Na}}=0.28, X_{\mathrm{Ca}}=0.07\right)$. Magnetite in this granite suggests a high oxidation state.

Amphibole in the mafic granulite enclave is magnesio-hornblende with marginal $\mathrm{Mg}$ enrichment $\left(X_{\mathrm{Mg}}=0.68_{\text {core }}\right.$ to $\left.0.7_{\text {rim }}\right)$ that is indicative of its prograde nature. Plagioclase is andesine; garnet is almandine-pyrope solid solution with small amount of the grossular component $\left(X_{\mathrm{Ca}}=0.16\right)$; clinopyroxene is diopsidehedenbergite solid solution with 0.78 diopside component.

\subsection{Granite $C$ (table 4)}

Perthitic feldspar has a potassic host $\left(X_{\mathrm{K}}=0.7\right)$ and sodic exsolved blebs $\left(X_{\mathrm{Na}}=0.73\right)$. Amphibole is potassic-magnesio hastingsite. Occasional andradite $\left(\mathrm{Fe}^{3}=1.37\right)$ and magnetite $\left(\mathrm{Fe}^{3}=1.99\right)$ are indicative of a high oxidation state.
In the amphibolite enclave amphibole is potassic hastingsite and has a more ferroan composition $\left(X_{\mathrm{Mg}}=0.19\right)$ than that in the host granite $\left(X_{\mathrm{Mg}}=0.28\right)$. Alkali feldspar is rich in sodium component $\left(X_{\mathrm{Na}}=0.76,0.97\right)$. Andradite garnet and magnetite are more common in the enclave and hence may have been present in the source rocks.

\subsection{Granite D (table 5)}

Garnet is mainly almandine-pyrope solid solution $\left(X_{\mathrm{alm}}=0.8 ; X_{\mathrm{pyr}}=0.16\right)$. Perthitic feldspar has a potassic host $\left(X_{\mathrm{K}}=0.89 ; X_{\mathrm{Na}}=0.1\right)$ and a more sodic exsolved component $\left(X_{\mathrm{Na}}=0.33\right)$. Biotite is phlogopite-annite solid solution, of intermediate composition $\left(X_{\mathrm{Mg}}=0.5\right)$. Included biotite in garnet is more magnesian $\left(X_{\mathrm{Mg}}=0.6\right)$ and has higher $\mathrm{TiO}_{2}(5.57 \%$ compared to $4.74 \%$ of the matrix biotite) and could be restitic.

In the metapelitic granulite enclave garnet is almandine-pyrope solid solution, but garnet in the enclave is more magnesian than that in the granite $\left(X_{\mathrm{Mg}}=0.37-0.38\right.$ against 0.16$)$, and this is consistent with the restitic nature of the enclave. Besides rutile, opaque oxide of hemo-ilmenite composition is indicative of low oxidation state.

\subsection{Granite E (table 6)}

Besides the microcline-type alkali feldspar (not analyzed), the feldspar with lamellar twinning is albitic plagioclase $\left(X_{\mathrm{Na}}=0.96 ; X_{\mathrm{Ca}}=0.03\right)$. Chlorite and epidote are both of ferroan composition. 

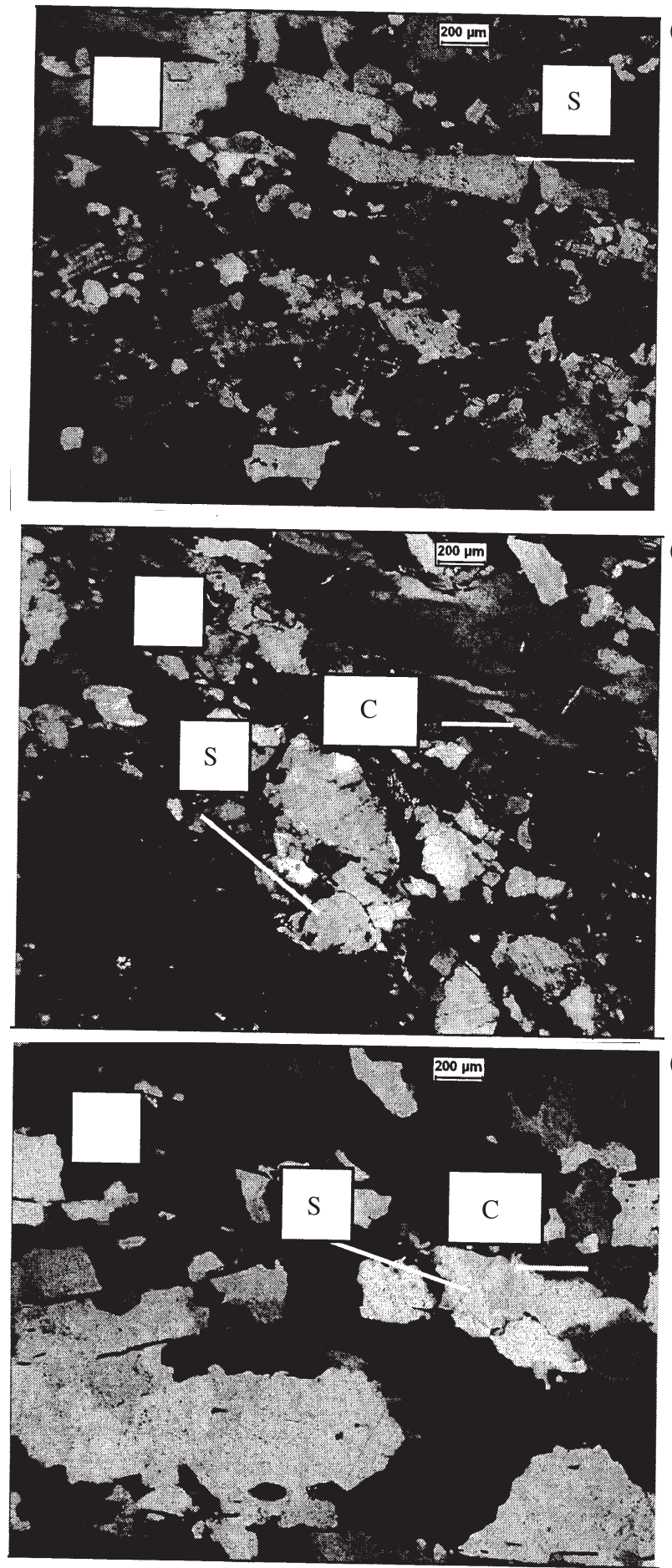

Figure 4. Photomicrographs. (a) Recrystallized quartz ribbons showing no effects of strain, presumably due to annealing at high-temperature. (b) Hornblende alignment, defining magmatic mineral foliation, is parallel to quartz ribbons, defining a solid-state mylonitic foliation. An incipient cleavage or C-fabric is also seen. (c) Aligned quartz-feldspar porphyroclasts define a mylonitic foliation, $\mathrm{S}$ and oblique fabric $\mathrm{C}$ is defined by mica fish.

The composition of the epidote suggests its magmatic nature (Tulloch 1986).

In the schistose enclave, beside the ferrochlorite, which is similar to that in the granite host
$\left(X_{\mathrm{Mg}}=0.37\right.$ against 0.35$)$, tremolitic amphibole is a ferrogedrite (calcium poor). Feldspar with lamellar twinning is albitic plagioclase, similar to that in the granite $\left(X_{\mathrm{Na}}=0.94 ; X_{\mathrm{Ca}}=0.05\right)$.

\section{Pressure-temperature record in the granulites of the adjacent EGMB}

Maximum pressure-temperature estimates could be recorded from two-pyroxene granulites, which occur as banded charnockitic gneiss, in the western part of the study area around Muktapusi. A post-peak decompression is recorded from metapelitic granulite xenoliths, occurring as enclaves in granite D. Compositions of coexisting mineral phases in these rocks are presented in tables 7 and 8. Geothermobarometric estimates were obtained using the internally consistent thermodynamic data base of Berman (1988). From core compositions of adjacent garnet and clinopyroxene, using the solution model of Berman (1990) for garnet, estimated temperatures are $780^{\circ} \mathrm{C}$ and $830^{\circ} \mathrm{C}$ for the two samples. $\mathrm{A} \pm 20^{\circ} \mathrm{C}$ precision may be assigned to these estimates (Berman 1991). Pressure estimates are obtained from garnet-orthopyroxene-plagioclase-quartz and garnet-clinopyroxene-plagioclase-quartz assemblages respectively. In view of the pyroxene compositions (Mg-rich), Mg-end member reactions are better suited in these assemblages. Also Newton's Al-avoidance model for plagioclase is unsuitable, because of the anorthite-rich compositions of plagioclase. Hence, the $\mathrm{P}_{\mathrm{Mg}}$ barometer is considered and the solution models of Berman (1990) are used. $\mathrm{P}_{\mathrm{Mg}}$ estimates for the two samples are $8.5 \mathrm{kbar}$ at $780^{\circ} \mathrm{C}$ and $8.5 \mathrm{kbar}$ at $830^{\circ} \mathrm{C}$ respectively. Additionally, rim-rim compositions of garnet-clinopyroxene pairs in these samples record about $100^{\circ} \mathrm{C}$ cooling at $8 \mathrm{kbar}$. The metapelitic granulite assemblage: garnet-sillimanite-ilmeniterutile-quartz is not suitable for temperature estimate; however, a pressure estimate is made from this assemblage using the GRAIL barometer and Berman (1990) calibration, which gives $7.6 \mathrm{kbar}$. It should be noted that these barometric estimates can be assigned a precision of $\pm 0.5 \mathrm{kbar}$ (Berman 1991). Thus a post-peak equilibration at $800^{\circ} \mathrm{C}$ and $8 \mathrm{kbar}$ and isobaric cooling $\sim 100^{\circ} \mathrm{C}$ at $8 \mathrm{kbar}$ is recorded from the granulites of the study area. Incidentally, coronal garnet on pyroxene is the common texture in these rocks and this is consistent with isobaric cooling and the sense of the reaction $\mathrm{Cpx}+\mathrm{Plg} \rightarrow$ Grt + Qtz. A postpeak isobaric cooling path has been recorded from many localities in the Eastern Ghats (Sengupta et al 1990; Dasgupta et al 1993; Mukhopadhyay and Bhattacharya 1997; Kar et al 2003). The 
Table 2 .

\begin{tabular}{|c|c|c|c|c|c|c|c|c|}
\hline \multicolumn{5}{|c|}{$\begin{array}{l}\text { Representative mineral compositions in Granite A; } \\
\text { Sample no. SN } 138\end{array}$} & \multicolumn{4}{|c|}{$\begin{array}{l}\text { Minerals in enclave; } \\
\text { Sample no. SN 29A }\end{array}$} \\
\hline Minerals & Amph.c & Amph.r & Feldspar & Epidote & Minerals Amph. & Feldspar & Feldspar & Ilmenite \\
\hline $\mathrm{SiO}_{2}$ & 40.13 & 40.85 & 67.13 & 38.91 & 44.09 & 67.71 & 64.32 & 0.66 \\
\hline $\mathrm{TiO}_{2}$ & 0.14 & 0.05 & 0.05 & 0.02 & 1.69 & & & 52.64 \\
\hline $\mathrm{Al}_{2} \mathrm{O}_{3}$ & 10.74 & 9.86 & 20.13 & 23.79 & 8.25 & 20.12 & 18.79 & \\
\hline $\mathrm{FeO}$ & 30.52 & 29.87 & & 13.22 & 28.23 & & & 41.6 \\
\hline $\mathrm{MnO}$ & 0.01 & 1.1 & & 0.18 & 0.48 & & & 3.31 \\
\hline $\mathrm{MgO}$ & 1.99 & 2.38 & & & 3.7 & & & 0.35 \\
\hline $\mathrm{CaO}$ & 9.89 & 9.85 & 0.12 & 21.38 & 10.15 & 0.29 & 0.35 & \\
\hline $\mathrm{Na}_{2} \mathrm{O}$ & 1.68 & 1.57 & 10.97 & 0.23 & 2.08 & 12.08 & 15.94 & \\
\hline $\mathrm{K}_{2} \mathrm{O}$ & 2.21 & 1.97 & 1.25 & 0.11 & 1.23 & 0.2 & & \\
\hline Total & 97.31 & 97.49 & 99.65 & 97.84 & 99.89 & 100.4 & 99.39 & 98.55 \\
\hline $\mathrm{O}$ in unit & 22 & 22 & 8 & 12 & 22 & 8 & 8 & 3 \\
\hline $\mathrm{Si}$ & 6.14 & 6.26 & 2.95 & 3.06 & 6.55 & 2.96 & 2.9 & 0.12 \\
\hline $\mathrm{Ti}$ & 0.02 & 0.01 & 0.002 & 0.01 & 0.19 & & & 1.001 \\
\hline $\mathrm{Al}$ & 1.94 & 1.78 & 1.04 & 2.21 & 1.44 & 1.04 & 1 & \\
\hline $\mathrm{Fe}^{2}$ & 3.78 & 3.74 & & 0.89 & 3.52 & & & 0.88 \\
\hline $\mathrm{Fe}^{3}$ & 0.12 & 0.09 & & & & & & \\
\hline $\mathrm{Mn}$ & 0.001 & 0.12 & & 0.01 & 0.05 & & & 0.07 \\
\hline $\mathrm{Mg}$ & 0.45 & 0.54 & & & 0.82 & & & 0.01 \\
\hline $\mathrm{Ca}$ & 1.62 & 1.62 & 0.006 & 1.8 & 1.61 & 0.01 & 0.02 & \\
\hline $\mathrm{Na}$ & 0.5 & 0.46 & 0.93 & 0.03 & 0.6 & 1.02 & 1.39 & \\
\hline $\mathrm{K}$ & 0.43 & 0.39 & 0.07 & & 0.23 & 0.01 & & \\
\hline Cat. Sum & 14.99 & 14.99 & 4.99 & 8.03 & 15.01 & 5.04 & 5.3 & 2.08 \\
\hline $\mathrm{X}_{\mathrm{Mg}}$ & 0.11 & 0.13 & & & 0.19 & & & \\
\hline $\mathrm{XCa}$ & & & 0.006 & & & 0.01 & 0.01 & \\
\hline $\mathrm{XNa}$ & & & 0.92 & & & 0.98 & 0.99 & \\
\hline $\mathrm{X}_{\mathrm{K}}$ & & & 0.07 & & & 0.01 & & \\
\hline
\end{tabular}

Table 3.

\begin{tabular}{|c|c|c|c|c|c|c|c|c|c|c|c|c|}
\hline \multicolumn{7}{|c|}{$\begin{array}{c}\text { Representative mineral compositions in Granite B; } \\
\text { Sample no. SN } 11 .\end{array}$} & \multicolumn{6}{|c|}{$\begin{array}{l}\text { Minerals in mafic granulite enclave; } \\
\text { Sample no. SN } 57\end{array}$} \\
\hline Minerals & Biot.c & Biot.r & Plag-fls & Alk-fls & Alk-fls & Mag. & & Garnet & $\mathrm{Cpx}$ & Amph.c & Amph.r & Plag. \\
\hline $\mathrm{SiO}_{2}$ & 36.45 & 36.04 & 63.05 & 65.49 & 66.55 & & & 38.25 & 51.67 & 45.75 & 46.04 & 59.02 \\
\hline $\mathrm{TiO}_{2}$ & 5.74 & 6.14 & & & & 0.07 & & 0.08 & 0.24 & 1.56 & 1.5 & 0.06 \\
\hline $\mathrm{Al}_{2} \mathrm{O}_{3}$ & 14.12 & 14.38 & 23.6 & 20.25 & 18.48 & 0.02 & & 23.14 & 3.19 & 11.41 & 11.66 & 26.35 \\
\hline $\mathrm{FeO}$ & 20.46 & 19.79 & & & & 90.73 & & 24.59 & 7.47 & 11.76 & 11.06 & 0.12 \\
\hline $\mathrm{MnO}$ & 0.18 & 0.14 & & & & 0.06 & & 0.82 & 0.05 & 0.13 & & \\
\hline $\mathrm{MgO}$ & 11.43 & 11.33 & & & & & & 8.14 & 14.88 & 14.37 & 14.9 & \\
\hline $\mathrm{CaO}$ & & 0.04 & 4.9 & 1.35 & 0.03 & & & 5.74 & 20.78 & 10.56 & 10.46 & 6.57 \\
\hline $\mathrm{Na}_{2} \mathrm{O}$ & & & 0.16 & 3.18 & 1.07 & & & 0.02 & 1.1 & 1.92 & 2 & 8.02 \\
\hline $\mathrm{K}_{2} \mathrm{O}$ & 9.82 & 9.87 & 8.14 & 11.21 & 15.82 & & & 0.03 & 0.04 & 0.31 & 0.25 & 0.12 \\
\hline Total & 98.21 & 97.72 & 99.85 & 101.5 & 101.84 & 90.87 & & 100.8 & 99.42 & 97.89 & 97.87 & 100.26 \\
\hline $\mathrm{O}$ in unit & 22 & 22 & 8 & 8 & 8 & 4 & & 12 & 6 & 22 & 22 & 8 \\
\hline $\mathrm{Si}$ & 5.45 & 5.4 & 2.89 & 2.94 & 3.02 & & & 2.91 & 1.9 & 6.39 & 6.4 & 2.61 \\
\hline $\mathrm{Ti}$ & 0.65 & 0.69 & & & & 0.002 & & 0.004 & 0.006 & 0.16 & 0.16 & 0.002 \\
\hline $\mathrm{Al}$ & 2.49 & 2.54 & 1.23 & 1.07 & 0.98 & 0.001 & & 2.08 & 0.14 & 1.88 & 1.91 & 1.37 \\
\hline $\mathrm{Fe}^{2}$ & 2.56 & 2.48 & & & & 1.005 & & 1.55 & 0.23 & 1.4 & 1.31 & 0.004 \\
\hline $\mathrm{Fe}^{3}$ & & & & & & 1.99 & & 0.12 & 0.004 & & & \\
\hline Mn & 0.02 & 0.02 & & & & 0.002 & & 0.04 & 0.001 & 0.01 & & \\
\hline $\mathrm{Mg}$ & 2.55 & 2.53 & & & & & & 0.92 & 0.82 & 3.01 & 3.09 & \\
\hline $\mathrm{Ca}$ & & 0.01 & 0.23 & 0.07 & 0.002 & & & 0.47 & 0.82 & 1.58 & 1.56 & 0.31 \\
\hline $\mathrm{Na}$ & & & 0.7 & 0.28 & 0.09 & & & 0.003 & 0.08 & 0.52 & 0.54 & 0.69 \\
\hline $\mathrm{K}$ & 1.87 & 1.89 & 0.01 & 0.64 & 0.91 & & & 0.003 & 0.002 & 0.06 & 0.04 & 0.006 \\
\hline Cat. Sum & 15.52 & 15.55 & 5.05 & 4.99 & 5.01 & 2.99 & & 8.1 & 3.99 & 15.01 & 15.01 & 4.99 \\
\hline $\mathrm{X}_{\mathrm{Mg}}$ & 0.49 & 0.51 & & & & & & 0.37 & 0.78 & 0.68 & 0.7 & \\
\hline $\mathrm{X}_{\mathrm{Ca}}$ & & & 0.25 & 0.07 & 0.002 & & Alm & 0.52 & & & & 0.31 \\
\hline $\mathrm{X}_{\mathrm{Na}}$ & & & 0.74 & 0.28 & 0.09 & & Pyr & 0.31 & & & & 0.69 \\
\hline $\mathrm{X}_{\mathrm{K}}$ & & & 0.01 & 0.65 & 0.9 & & $\mathrm{Gr}$ & 0.16 & & & & \\
\hline
\end{tabular}


Table 4

\begin{tabular}{|c|c|c|c|c|c|c|c|c|c|c|}
\hline \multicolumn{6}{|c|}{$\begin{array}{c}\text { Representative mineral compositions in Granite C; } \\
\text { Sample no. SD 52A }\end{array}$} & \multicolumn{5}{|c|}{$\begin{array}{l}\text { Minerals in Amphibolite enclave; } \\
\text { Sample no. SD 52C }\end{array}$} \\
\hline Minerals & Perth-h & Perth-b & Amph. & Garnet & Magnetite & Alk-fls & Alk-fls & Amph. & Garnet & Magnetite \\
\hline $\mathrm{SiO}_{2}$ & 66.89 & 69.53 & 40.99 & 36.66 & & 70.64 & 67.84 & 38.88 & 36.5 & 0.05 \\
\hline $\mathrm{TiO}_{2}$ & & & 0.13 & 0.4 & & & & 0.09 & 0.17 & 0.09 \\
\hline $\mathrm{Al}_{2} \mathrm{O}_{3}$ & 18.69 & 19.34 & 10.15 & 6.39 & & 18.12 & 19.33 & 11.59 & 8.13 & 0.02 \\
\hline $\mathrm{FeO}$ & & & 23.99 & 23.07 & 89.32 & & & 25.51 & 22.11 & 88.13 \\
\hline $\mathrm{MnO}$ & & & 1.1 & 3.28 & 0.12 & & & 1.24 & 3.84 & 0.09 \\
\hline $\mathrm{MgO}$ & & & 5.74 & & & & & 3.27 & & 0.02 \\
\hline $\mathrm{CaO}$ & 0.09 & 0.22 & 10.67 & 27.03 & & 0.51 & 0.14 & 10.34 & 26.95 & \\
\hline $\mathrm{Na}_{2} \mathrm{O}$ & 3.04 & 7.79 & 1.83 & & & 10.66 & 8.1 & 1.43 & & \\
\hline $\mathrm{K}_{2} \mathrm{O}$ & 11.15 & 4.38 & 1.63 & & & 0.09 & 3.75 & 2.07 & & \\
\hline Total & 99.86 & 101.32 & 96.1 & 96.82 & 89.36 & 100.02 & 99.16 & 94.4 & 97.6 & 88.32 \\
\hline $\mathrm{O}$ in unit & 8 & 8 & 22 & 12 & 4 & 8 & 8 & 22 & 12 & 4 \\
\hline $\mathrm{Si}$ & 3.02 & 3.03 & 6.6 & 3.08 & & 3.07 & 3.01 & 6.06 & 3.03 & 0.002 \\
\hline $\mathrm{Ti}$ & & & 0.02 & 0.03 & & & & 0.01 & 0.01 & 0.002 \\
\hline $\mathrm{Al}$ & 1 & 0.99 & 1.92 & 0.63 & & 0.93 & 1.01 & 2.13 & 0.8 & 0.001 \\
\hline $\mathrm{Fe}^{2}$ & & & 3.54 & 0.25 & 0.99 & & & 3.24 & 0.34 & 1.01 \\
\hline $\mathrm{Fe}^{3}$ & & & & 1.37 & 1.99 & & & 0.09 & 1.2 & 1.97 \\
\hline $\mathrm{Mn}$ & & & 0.12 & 0.19 & 0.003 & & & 0.13 & 0.22 & 0.003 \\
\hline $\mathrm{Mg}$ & & & 1.38 & & & & & 0.76 & & \\
\hline $\mathrm{Ca}$ & 0.004 & 0.01 & 1.84 & 2.43 & & 0.02 & 0.01 & 1.73 & 2.4 & \\
\hline $\mathrm{Na}$ & 0.27 & 0.66 & 0.57 & & & 0.9 & 0.7 & 0.43 & & \\
\hline K & 0.64 & 0.24 & 0.34 & & & 0.01 & 0.21 & 0.41 & & \\
\hline Cat. Sum & 4.93 & 4.93 & 15 & 7.98 & 3 & 4.93 & 4.94 & 15 & 7.99 & 2.99 \\
\hline
\end{tabular}

Table 5 .

\begin{tabular}{|c|c|c|c|c|c|c|c|c|c|c|}
\hline \multicolumn{6}{|c|}{$\begin{array}{c}\text { Representative mineral compositions in Granite D; } \\
\text { Sample no. SD } 9 / 10\end{array}$} & \multicolumn{5}{|c|}{$\begin{array}{l}\text { Minerals in metapelitic enclave; } \\
\text { Sample no. } 20 \mathrm{~A}\end{array}$} \\
\hline Minerals & Garnet & Perth-h & Perth-b & Biot. & Biot. incl. & & Grt-core & Grt-rim & Crd-core & Crd-rim \\
\hline $\mathrm{SiO}_{2}$ & 37.89 & 66.22 & 65.56 & 38.09 & 36.83 & $\mathrm{SiO}_{2}$ & 39.91 & 39.73 & 50.48 & 50.53 \\
\hline $\mathrm{TiO}_{2}$ & 0.006 & & & 4.74 & 5.57 & $\mathrm{TiO}_{2}$ & & 0.02 & & \\
\hline $\mathrm{Al}_{2} \mathrm{O}_{3}$ & 21.45 & 18.09 & 19.77 & 19.96 & 16.46 & $\mathrm{Al}_{2} \mathrm{O}_{3}$ & 22.75 & 22.36 & 34.14 & 34.3 \\
\hline $\mathrm{FeO}$ & 35.66 & & & 15.03 & 15.33 & $\mathrm{FeO}$ & 26.88 & 27.55 & 3.59 & 3.58 \\
\hline $\mathrm{MnO}$ & 1.25 & & & 0.02 & 0.03 & $\mathrm{MnO}$ & 0.6 & 0.46 & 0.004 & 0.02 \\
\hline $\mathrm{MgO}$ & 4.1 & & & 8.68 & 12.68 & $\mathrm{MgO}$ & 9.48 & 9.87 & 12.41 & 13.04 \\
\hline $\mathrm{CaO}$ & 0.8 & 0.12 & 0.9 & 0.07 & 0.02 & $\mathrm{CaO}$ & 0.86 & 0.63 & 0.1 & 0.03 \\
\hline $\mathrm{Na}_{2} \mathrm{O}$ & & 0.99 & 3.68 & 0.08 & 0.06 & $\mathrm{Na}_{2} \mathrm{O}$ & & & 0.06 & \\
\hline $\mathrm{K}_{2} \mathrm{O}$ & & 13.35 & 10.79 & 8.81 & 9.94 & $\mathrm{~K}_{2} \mathrm{O}$ & & & 0.02 & 0.006 \\
\hline Total & 101.1 & 98.76 & 100.68 & 95.35 & 96.92 & Total & 100.47 & 100.55 & 100.5 & 100.68 \\
\hline $\mathrm{O}$ in unit & 12 & 8 & 8 & 22 & 22 & $\mathrm{O}$ in unit & 12 & 12 & 18 & 18 \\
\hline $\mathrm{Si}$ & 3.01 & 3.04 & 2.95 & 5.59 & 5.42 & $\mathrm{Si}$ & 3.05 & 3.02 & 4.93 & 4.9 \\
\hline $\mathrm{Ti}$ & & & & 0.52 & 0.62 & $\mathrm{Ti}$ & & & & \\
\hline $\mathrm{Al}$ & 2.01 & 0.98 & 1.05 & 3.45 & 2.85 & $\mathrm{Al}$ & 2.05 & 2.01 & 3.93 & 3.92 \\
\hline $\mathrm{Fe}^{2}$ & 2.37 & & & 1.88 & 1.89 & $\mathrm{Fe}^{2}$ & 1.74 & 1.77 & 0.29 & 0.29 \\
\hline $\mathrm{Fe}^{3}$ & & & & & & $\mathrm{Fe}^{3}$ & & & 0.003 & 0.004 \\
\hline $\mathrm{Mn}$ & 0.07 & & & 0.002 & 0.003 & Mn & 0.03 & 0.02 & 0.001 & 0.001 \\
\hline $\mathrm{Mg}$ & 0.48 & & & 1.9 & 2.78 & $\mathrm{Mg}$ & 1.08 & 1.12 & 1.81 & 1.88 \\
\hline $\mathrm{Ca}$ & 0.07 & 0.01 & 0.04 & 0.01 & 0.003 & $\mathrm{Ca}$ & 0.07 & 0.05 & 0.01 & 0.003 \\
\hline $\mathrm{Na}$ & & 0.09 & 0.32 & 0.02 & 0.02 & $\mathrm{Na}$ & & & 0.01 & \\
\hline $\mathrm{K}$ & & 0.78 & 0.62 & 1.65 & 1.86 & $\mathrm{~K}$ & & & 0.002 & \\
\hline Cat. Sum & 8 & 4.9 & 4.98 & 15.02 & 15.45 & Cat. Sum & 8.02 & 8.01 & 11 & 11 \\
\hline $\mathrm{X}_{\mathrm{Mg}}$ & 0.16 & & & 0.5 & 0.6 & $\mathrm{X}_{\mathrm{Mg}}$ & 0.37 & 0.38 & 0.85 & 0.99 \\
\hline $\mathrm{X}_{\mathrm{Ca}}$ & 0.02 & 0.01 & 0.04 & & & & & & & \\
\hline $\mathrm{X}_{\mathrm{Na}}$ & & 0.1 & 0.33 & & & & & & & \\
\hline $\mathrm{X}_{\mathrm{K}}$ & & 0.89 & 0.63 & & & & & & & \\
\hline
\end{tabular}


Table 6.

\begin{tabular}{|c|c|c|c|c|c|c|}
\hline \multicolumn{4}{|c|}{$\begin{array}{c}\text { Representative mineral compositions in Granite E: } \\
\text { Sample no. SD 34B }\end{array}$} & \multicolumn{3}{|c|}{$\begin{array}{l}\text { Minerals in Chlorite schist enclave: } \\
\text { Sample no. } 16 / 86 \mathrm{~A}\end{array}$} \\
\hline Minerals & Epidote & Chlorite & Feldspar & Chlorite & Amph. & Feldspar \\
\hline $\mathrm{SiO}_{2}$ & 36.5 & 24.55 & 67.98 & 25.22 & 33.48 & 66.79 \\
\hline $\mathrm{TiO}_{2}$ & 0.12 & 0.02 & & 0.02 & 0.04 & \\
\hline $\mathrm{Al}_{2} \mathrm{O}_{3}$ & 24.87 & 20.17 & 19.44 & 20.81 & 18.47 & 20 \\
\hline $\mathrm{FeO}$ & 8.1 & 33.36 & & 30.84 & 28.13 & \\
\hline $\mathrm{MnO}$ & 0.14 & 0.68 & & 0.49 & 0.49 & \\
\hline $\mathrm{MgO}$ & 0.05 & 9.95 & & 10.15 & 7.94 & \\
\hline $\mathrm{CaO}$ & 23.19 & 0.02 & 0.57 & 0.03 & 0.31 & 0.82 \\
\hline $\mathrm{Na}_{2} \mathrm{O}$ & & & 10.22 & 0.41 & & 9.4 \\
\hline $\mathrm{K}_{2} \mathrm{O}$ & & 0.05 & 0.05 & 0.15 & 0.002 & 0.13 \\
\hline Total & 92.21 & 88.6 & 98.9 & 88 & 88.8 & 97.12 \\
\hline $\mathrm{O}$ in unit & 12 & 28 & 8 & 28 & 24 & 8 \\
\hline $\mathrm{Si}$ & 2.92 & 5.36 & 3 & 5.47 & 5.87 & 2.99 \\
\hline $\mathrm{Ti}$ & 0.01 & 0.003 & & 0.003 & 0.01 & \\
\hline $\mathrm{Al}$ & 2.34 & 5.19 & 1.01 & 5.32 & 3.82 & 1.05 \\
\hline $\mathrm{Fe}^{2}$ & 0.54 & 6.09 & & 5.59 & 4.74 & \\
\hline \multicolumn{7}{|l|}{$\mathrm{Fe}^{3}$} \\
\hline $\mathrm{Mn}$ & 0.01 & 0.13 & & 0.09 & 0.06 & \\
\hline $\mathrm{Mg}$ & 0.01 & 3.24 & & 3.28 & 2.07 & \\
\hline $\mathrm{Ca}$ & 1.99 & 0.01 & 0.03 & 0.01 & 0.06 & 0.04 \\
\hline $\mathrm{Na}$ & & & 0.88 & 0.17 & & 0.82 \\
\hline $\mathrm{K}$ & & 0.01 & 0.003 & 0.04 & & 0.01 \\
\hline Cat. Sum & 7.82 & 20.03 & 4.93 & 19.95 & 16.4 & 4.9 \\
\hline $\mathrm{X}_{\mathrm{Mg}}$ & $\mathrm{X}_{\text {Pist }}=0.17$ & 0.35 & & 0.37 & Ferro & Albite \\
\hline $\mathrm{X}_{\mathrm{Ca}}$ & \multicolumn{2}{|c|}{ Ferro } & 0.03 & Ferro & \multirow[t]{3}{*}{ Gedrite } & 0.05 \\
\hline $\mathrm{X}_{\mathrm{Na}}$ & \multirow{2}{*}{\multicolumn{2}{|c|}{ Chlorite }} & 0.96 & Chlorite & & 0.94 \\
\hline $\mathrm{X}_{\mathrm{K}}$ & & & & & & \\
\hline
\end{tabular}

Table 7. Composition of coexisting phases for pressure-temperature estimates.

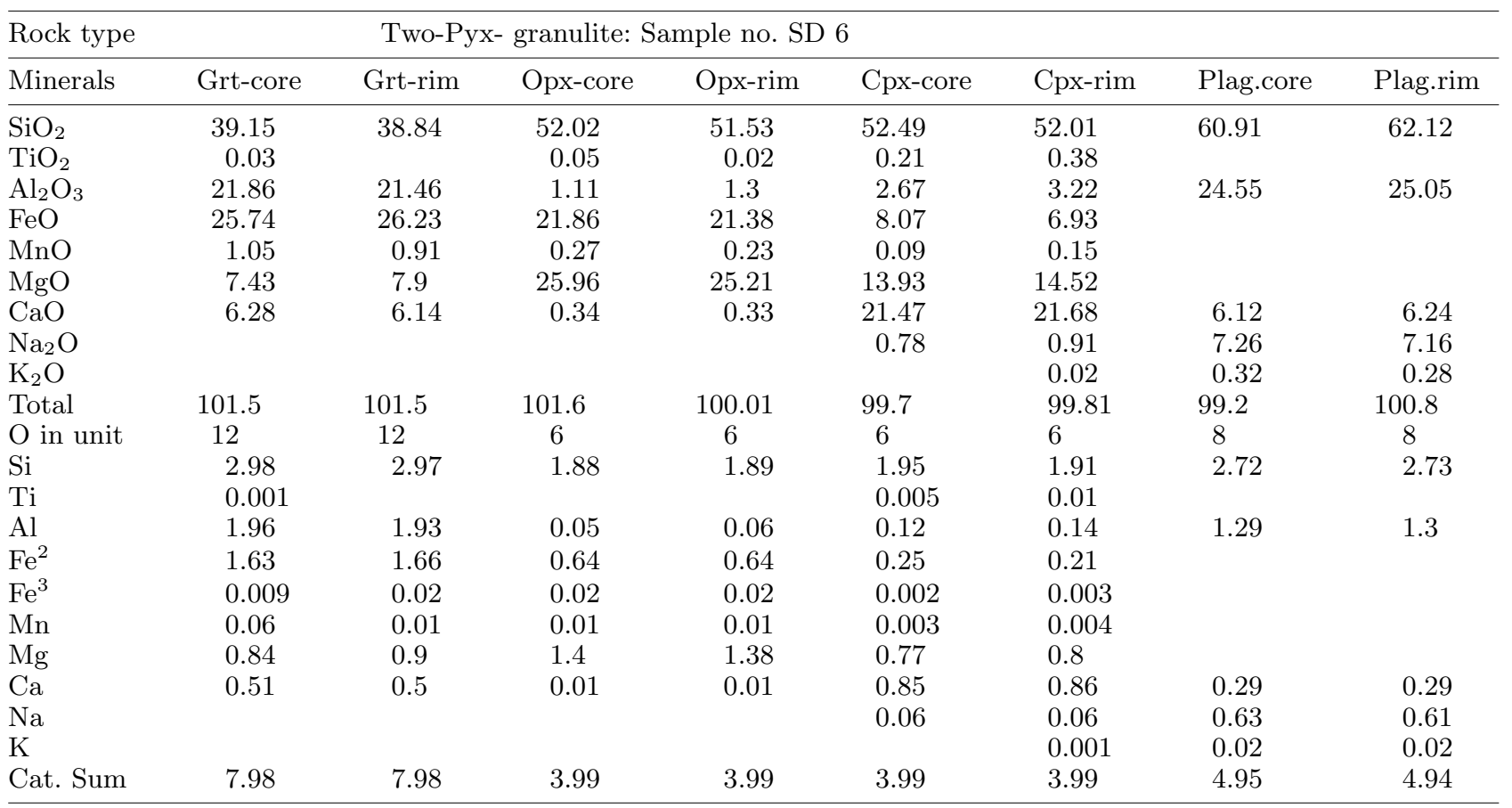

Berman (1990) calibration for core compositions of garnet and orthopyroxene gives $780^{\circ} \mathrm{C}$.

$\mathrm{P}_{\mathrm{Mg}}$ estimate from garnet-clinopyroxene-plagioclase-quartz assemblage, using Berman (1990).

Calibration gives $8.5 \mathrm{kbar}$ at $780^{\circ} \mathrm{C}$. Rim-Rim compositions indicate a cooling $\sim 100^{\circ} \mathrm{C}$ at $8 \mathrm{kbar}$. 
Table 7. (Continued).

\begin{tabular}{|c|c|c|c|c|c|}
\hline \multicolumn{6}{|c|}{ Minerals in mafic granulite: Sample no. SN 57} \\
\hline Minerals & Garnet & Cpx & Amph.c & Amph.r & Plag. \\
\hline $\mathrm{SiO}_{2}$ & 38.25 & 51.67 & 45.75 & 46.04 & 59.02 \\
\hline $\mathrm{TiO}_{2}$ & 0.08 & 0.24 & 1.56 & 1.5 & 0.06 \\
\hline $\mathrm{Al}_{2} \mathrm{O}_{3}$ & 23.14 & 3.19 & 11.41 & 11.66 & 26.35 \\
\hline $\mathrm{FeO}$ & 24.59 & 7.47 & 11.76 & 11.06 & 0.12 \\
\hline $\mathrm{MnO}$ & 0.82 & 0.05 & \multicolumn{2}{|l|}{0.13} & \\
\hline $\mathrm{MgO}$ & 8.14 & 14.88 & 14.37 & 14.9 & \\
\hline $\mathrm{CaO}$ & 5.74 & 20.78 & 10.56 & 10.46 & 6.57 \\
\hline $\mathrm{Na}_{2} \mathrm{O}$ & 0.02 & 1.1 & 1.92 & 2 & 8.02 \\
\hline $\mathrm{K}_{2} \mathrm{O}$ & 0.03 & 0.04 & 0.31 & 0.25 & 0.12 \\
\hline Total & 100.8 & 99.42 & 97.89 & 97.87 & 100.26 \\
\hline $\mathrm{O}$ in unit & 12 & 6 & 22 & 22 & 8 \\
\hline $\mathrm{Si}$ & 2.91 & 1.9 & 6.39 & 6.4 & 2.61 \\
\hline $\mathrm{Ti}$ & 0.004 & 0.006 & 0.16 & 0.16 & 0.002 \\
\hline $\mathrm{Al}$ & 2.08 & 0.14 & 1.88 & 1.91 & 1.37 \\
\hline $\mathrm{Fe}^{2}$ & 1.55 & 0.23 & \multirow[t]{2}{*}{1.4} & \multirow[t]{2}{*}{1.31} & \multirow[t]{2}{*}{0.004} \\
\hline $\mathrm{Fe}^{3}$ & 0.12 & 0.004 & & & \\
\hline Mn & 0.04 & 0.001 & \multicolumn{2}{|l|}{0.01} & \\
\hline $\mathrm{Mg}$ & 0.92 & 0.82 & 3.01 & \multicolumn{2}{|l|}{309} \\
\hline $\mathrm{Ca}$ & 0.47 & 0.82 & 1.58 & 1.56 & 0.31 \\
\hline $\mathrm{Na}$ & 0.003 & 0.08 & 0.52 & 0.54 & 0.69 \\
\hline $\mathrm{K}$ & 0.003 & 0.002 & 0.06 & 0.04 & 0.006 \\
\hline Cat. Sum & 8.1 & 3.99 & & 15.01 & \multirow[t]{2}{*}{4.99} \\
\hline $\mathrm{X}_{\mathrm{Mg}}$ & 0.37 & 0.78 & $\begin{array}{r}15.01 \\
0.68\end{array}$ & 0.7 & \\
\hline Garnet/cli & ene core & sitions & $0^{\circ} \mathrm{C}$ & & \\
\hline $\begin{array}{l}\mathrm{P}_{\mathrm{Mg}} \text { gives } \\
\text { calibration }\end{array}$ & at $830^{\circ}$ & g Berm & 90) & & \\
\hline
\end{tabular}

decompression reaction: garnet + sillimanite + quartz $\rightarrow$ cordierite, indicated by cordierite growth at garnet margin, with relict sillimanite in the matrix, was recorded from a metapelitic granulite enclave. Pressure-temperature estimates, for this assemblage, using Bhattacharya's (1993) calibrations, of $600^{\circ} \mathrm{C}$ and $4.8 \mathrm{kbar}$ are obtained. However, it is noted that garnet compositions in the metapelitic enclave in Granite D are different from those in the common metapelitic granulite assemblage; more magnesian garnet $\left(X_{\mathrm{Mg}}=0.37\right)$ in the former than in the latter $\left(X_{\mathrm{Mg}}=0.23\right)$. These apparently contrasting signatures, decompression at lower temperatures, but with a more magnesian garnet composition, should imply two unrelated metamorphic imprints, namely a post-peak isobaric cooling, recorded in the granulites, and a decompression, recorded only in a metapelitic enclave (figure 5). Presence of inclusions of biotite and sillimanite within garnet in Granite D, indicating a pelitic source; more magnesian garnet in the enclave compared to that in the host Granite $\mathrm{D}$; are consistent with the restitic nature of the enclave, as observed in the melting experiments (Le Breton and Thompson 1988). Hence, this decompression could only be related to emplacement of the granite (along with the enclaves) at higher crustal levels. In other words, the decompressional P-T path may relate to the collisional juxta position of the granulite belt against the Singhbhum craton to the north (Bhattacharya 1997).

\section{Rock chemistry}

Major- and trace-element analyses by XRF spectrometry were carried out at the Wadia Institute of Himalayan Geology, Dehradun. Operating condition for XRF machine was $20 / 40 \mathrm{kV}$ for major oxides and $55 / 60 \mathrm{kV}$ for trace elements. Nominal analysis time was 300 seconds for all major oxides and 100 seconds for each trace element. For the XRF analyses the overall accuracy (\% relative standard deviation) for major and minor oxides is better than $5 \%$ and for trace elements is better than $12 \%$. The average precision is reported as better than $1.5 \%$.

In tables 9 to 13 we present the analytical data for 25 granite samples, 5 from each of the 5 granitoid bodies. Two additional rocks, representing a mafic granulite enclave in Granite B and a metapelitic granulite enclave in Granite D were also analyzed and presented in the corresponding tables.

\subsection{Granite A}

These are high-silica (>72\%) granites (figure 6 ) and are marginally undersaturated with respect to 
Table 8. Composition of coexisting phases.

\begin{tabular}{|c|c|c|c|c|c|c|c|c|c|}
\hline \multirow{2}{*}{$\frac{\text { Rock type }}{\text { Minerals }}$} & \multicolumn{2}{|c|}{ Metapelite enclave } & \multicolumn{2}{|c|}{ Sample no. SD 20A } & \multirow{2}{*}{$\frac{\text { Metapelite }}{\text { Minerals }}$} & \multirow{2}{*}{$\begin{array}{l}\text { Sample } \\
\text { Garnet-c }\end{array}$} & \multicolumn{3}{|l|}{$26 / 56 \mathrm{~A}$} \\
\hline & Grt-core & Grt-rim & Crd-core & Crd-rim & & & Garnet-r & Hemo-Ilm & Rutile \\
\hline $\mathrm{SiO}_{2}$ & 39.91 & 39.73 & 50.48 & 50.53 & $\mathrm{SiO}_{2}$ & 38.78 & 39.12 & 1.04 & 0.01 \\
\hline $\mathrm{TiO}_{2}$ & & 0.02 & & & $\mathrm{TiO}_{2}$ & & & 65.19 & 101.02 \\
\hline $\mathrm{Al}_{2} \mathrm{O}_{3}$ & 22.75 & 22.36 & 34.14 & 34.3 & $\mathrm{Al}_{2} \mathrm{O}_{3}$ & 21.86 & 20.71 & 0.41 & 0.75 \\
\hline $\mathrm{FeO}$ & 26.88 & 27.55 & 3.59 & 3.58 & $\mathrm{FeO}$ & 32.74 & 31.63 & 20.9 & 0.15 \\
\hline $\mathrm{MnO}$ & 0.6 & 0.46 & 0.004 & 0.02 & $\mathrm{MnO}$ & 1.14 & 1.04 & 0.17 & \\
\hline $\mathrm{MgO}$ & 9.48 & 9.87 & 12.41 & 13.04 & $\mathrm{MgO}$ & 4.88 & 5.72 & 0.01 & \\
\hline $\mathrm{CaO}$ & 0.86 & 0.63 & 0.1 & 0.03 & $\mathrm{CaO}$ & 0.72 & 0.76 & & \\
\hline $\mathrm{Na}_{2} \mathrm{O}$ & & & 0.06 & & $\mathrm{Na}_{2} \mathrm{O}$ & & & & \\
\hline $\mathrm{K}_{2} \mathrm{O}$ & & & 0.02 & 0.006 & $\mathrm{~K}_{2} \mathrm{O}$ & & & & \\
\hline Total & 100.47 & 100.55 & 100.5 & 100.68 & Total & 100.1 & 98.88 & 87.71 & 101.93 \\
\hline $\mathrm{O}$ in unit & 12 & 12 & 18 & 18 & $\mathrm{O}$ in unit & 12 & 12 & 3 & 2 \\
\hline $\mathrm{Si}$ & 3.05 & 3.02 & 4.93 & 4.9 & $\mathrm{Si}$ & 3.08 & 3.12 & 0.03 & 0.001 \\
\hline $\mathrm{Ti}$ & & & & & $\mathrm{Ti}$ & & & 1.23 & 0.98 \\
\hline $\mathrm{Al}$ & 2.05 & 2.01 & 3.93 & 3.92 & $\mathrm{Al}$ & 2.04 & 1.95 & 0.01 & 0.01 \\
\hline $\mathrm{Fe}^{2}$ & 1.74 & 1.77 & 0.29 & 0.29 & $\mathrm{Fe}^{2}$ & 2.21 & 2.15 & 0.44 & 0.001 \\
\hline $\mathrm{Fe}^{3}$ & & & 0.003 & 0.004 & $\mathrm{Fe}^{3}$ & & & & \\
\hline Mn & 0.03 & 0.02 & 0.001 & 0.001 & Mn & 0.06 & 0.06 & 0.004 & \\
\hline $\mathrm{Mg}$ & 1.08 & 1.12 & 1.81 & 1.88 & $\mathrm{Mg}$ & 0.58 & 0.68 & 0.001 & \\
\hline $\mathrm{Ca}$ & 0.07 & 0.05 & 0.01 & 0.003 & $\mathrm{Ca}$ & 0.06 & 0.07 & & \\
\hline $\mathrm{Na}$ & & & 0.01 & & $\mathrm{Na}$ & & & & \\
\hline $\mathrm{K}$ & & & 0.002 & & $\mathrm{~K}$ & & & & \\
\hline Cat. Sum & 8.02 & 8.01 & 11 & 11 & Cat. Sum & 8.02 & 8.01 & 1.715 & 0.99 \\
\hline $\mathrm{X}_{\mathrm{Mg}}$ & 0.37 & 0.38 & 0.85 & 0.99 & $\mathrm{X}_{\mathrm{Mg}}$ & 0.2 & 0.23 & & \\
\hline
\end{tabular}

Garnet-cordierite-sillimanite-plagioclase-quartz assemblage using Bhattacharya (1993) calibration gives $600^{\circ} \mathrm{C}$ and $4.8 \mathrm{kbar}$.
Table 8. (Continued)

GRAIL barometer using Berman (1990) calibration gives $7.6 \mathrm{kbar}$.

\section{P-T path}

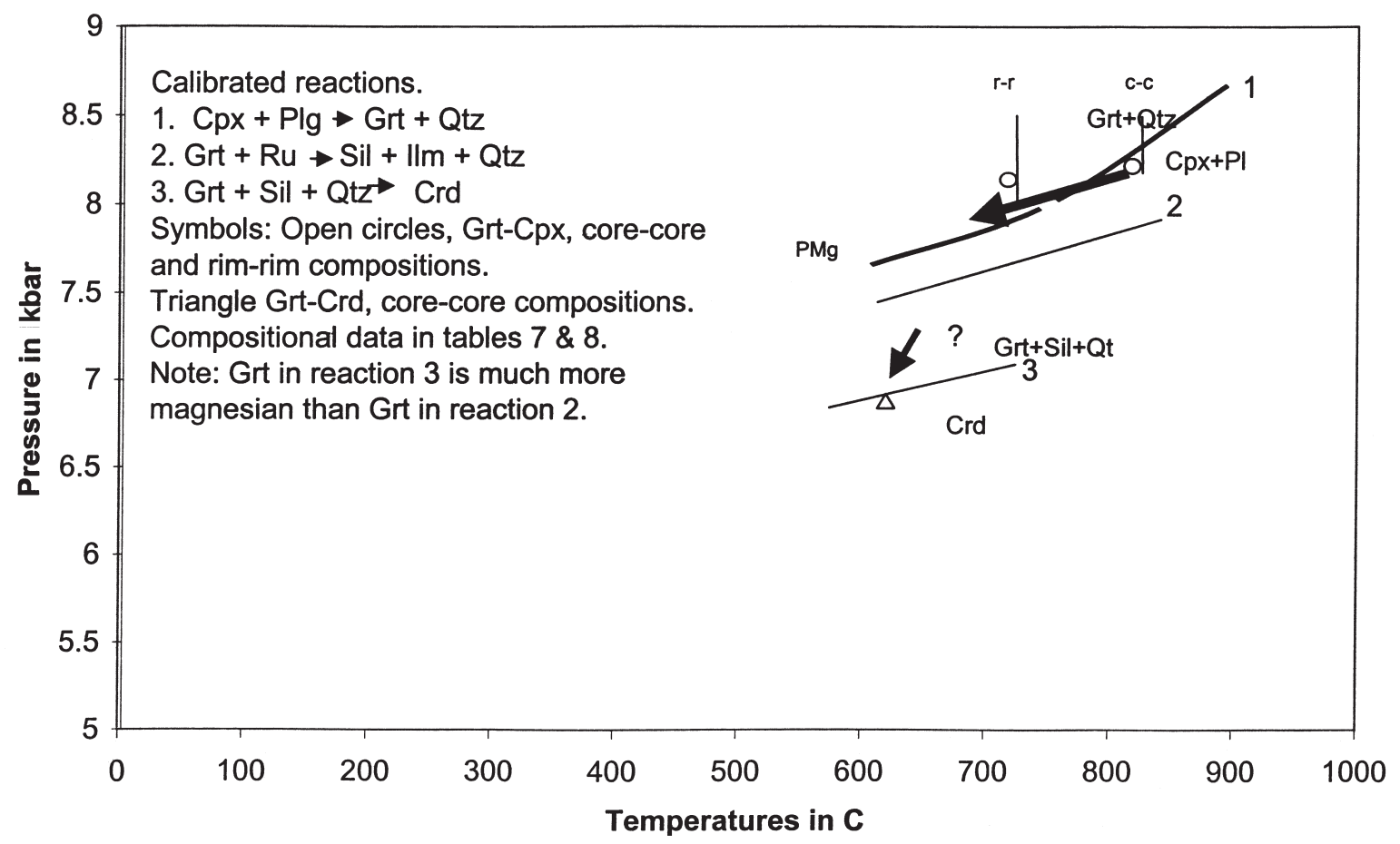

Figure 5. Pressure-temperature record and the P-T path derived from the granulites of the study area. 


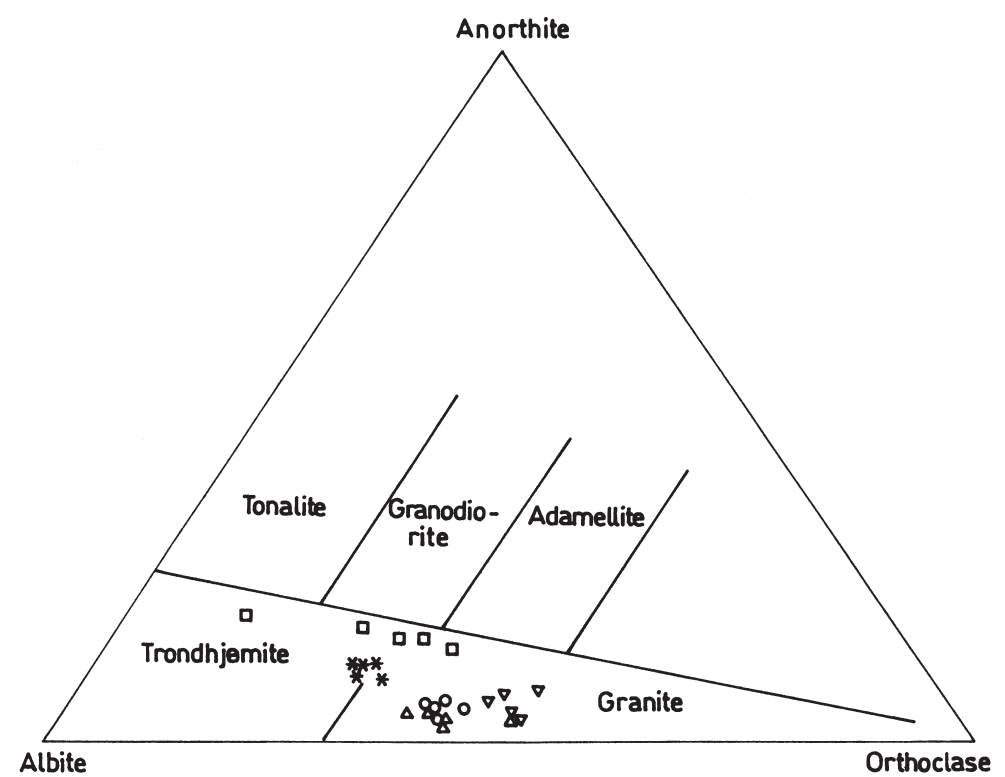

Figure 6. Normative plot of the granitoids, classified according to O'Connor (1965). Symbols: Circle = A, Square = B, Triangle $=\mathrm{C}$, Inverted triangle $=\mathrm{D}$, Asterix $=\mathrm{E}$.

Table 9. Bulk composition of Granite A.

\begin{tabular}{|c|c|c|c|c|c|}
\hline $\begin{array}{l}\text { Sample } \\
\text { Ref. no. }\end{array}$ & $\begin{array}{c}\text { S8/41 } \\
1\end{array}$ & $\begin{array}{l}\mathrm{S} 8 / 44 \\
2\end{array}$ & $\begin{array}{c}\text { SN106 } \\
3\end{array}$ & $\begin{array}{c}\text { SN32 } \\
4\end{array}$ & $\begin{array}{c}\text { SN32A } \\
5\end{array}$ \\
\hline $\mathrm{SiO}_{2}$ & 74.59 & 73.81 & 72.75 & 72.66 & 73.07 \\
\hline $\mathrm{TiO}_{2}$ & 0.33 & 0.35 & 0.36 & 0.39 & 0.39 \\
\hline $\mathrm{Al}_{2} \mathrm{O}_{3}$ & 11.76 & 11.94 & 12.02 & 12.03 & 11.8 \\
\hline $\mathrm{Fe}_{2} \mathrm{O}_{3}$ & 4.11 & 4.37 & 4.83 & 4.6 & 4.7 \\
\hline $\mathrm{MnO}$ & 0.08 & 0.07 & 0.07 & 0.09 & 0.08 \\
\hline $\mathrm{MgO}$ & 0.16 & 0.19 & 0.29 & 0.23 & 0.37 \\
\hline $\mathrm{CaO}$ & 1.23 & 1.18 & 1.31 & 1.37 & 1.26 \\
\hline $\mathrm{Na}_{2} \mathrm{O}$ & 3.56 & 3.81 & 3.86 & 3.69 & 3.83 \\
\hline $\mathrm{K}_{2} \mathrm{O}$ & 4.48 & 4.12 & 4.08 & 4.26 & 4.31 \\
\hline $\mathrm{P}_{2} \mathrm{O}_{5}$ & 0.11 & 0.11 & 0.11 & 0.14 & 0.15 \\
\hline LOI & 0.06 & 0.48 & 1.02 & 0.35 & 0.2 \\
\hline Total & 100.47 & 100.43 & 100.7 & 99.81 & 100.16 \\
\hline $\mathrm{A} / \mathrm{CNK}$ & 0.9 & 0.92 & 0.91 & 0.91 & 0.89 \\
\hline A/NK & 1.09 & 1.11 & 1.11 & 1.12 & 1.07 \\
\hline $\mathrm{K} / \mathrm{Na}$ & 1.4 & 1.2 & 1.2 & 1.3 & 1.3 \\
\hline Mg. no. & 7.08 & 7.85 & 10.52 & 8.92 & 13.34 \\
\hline \multicolumn{6}{|c|}{ Trace elements in ppm } \\
\hline $\mathrm{Ba}$ & 921 & 1056 & 1008 & 970 & 911 \\
\hline $\mathrm{Rb}$ & 162 & 76 & 153 & 134 & 162 \\
\hline $\mathrm{Sr}$ & 80 & 82 & 98 & 105 & 78 \\
\hline $\mathrm{Y}$ & 101 & 102 & 71 & 93 & 102 \\
\hline $\mathrm{Nb}$ & 34 & 38 & 36 & 36 & 38 \\
\hline $\mathrm{Zr}$ & 666 & 665 & 621 & 618 & 648 \\
\hline $\mathrm{Th}$ & 32 & 31 & 26 & 33 & 34 \\
\hline $\mathrm{U}$ & 8 & 2 & 7 & 6 & 8 \\
\hline $\mathrm{Pb}$ & 40 & 39 & 36 & 26 & 21 \\
\hline $\mathrm{Ga}$ & 19 & 21 & 20 & 21 & 18 \\
\hline $\mathrm{Ni}$ & 31 & 19 & 38 & 35 & 37 \\
\hline $\mathrm{Cu}$ & 9 & 8 & 10 & 7 & 7 \\
\hline $\mathrm{Zn}$ & 90 & 154 & 85 & 56 & 85 \\
\hline $\mathrm{Rb} / \mathrm{Sr}$ & 2.03 & 0.93 & 1.56 & 1.28 & 2.08 \\
\hline $\mathrm{Sr} / \mathrm{Y}$ & 0.8 & 0.8 & 1.38 & 1.12 & 0.8 \\
\hline
\end{tabular}

${ }^{*} \mathrm{Rb} / \mathrm{Sr}>1.0$, except $\mathrm{S} 8 / 44$.

${ }^{*} \mathrm{Sr} / \mathrm{Y} \sim 1.0$. 

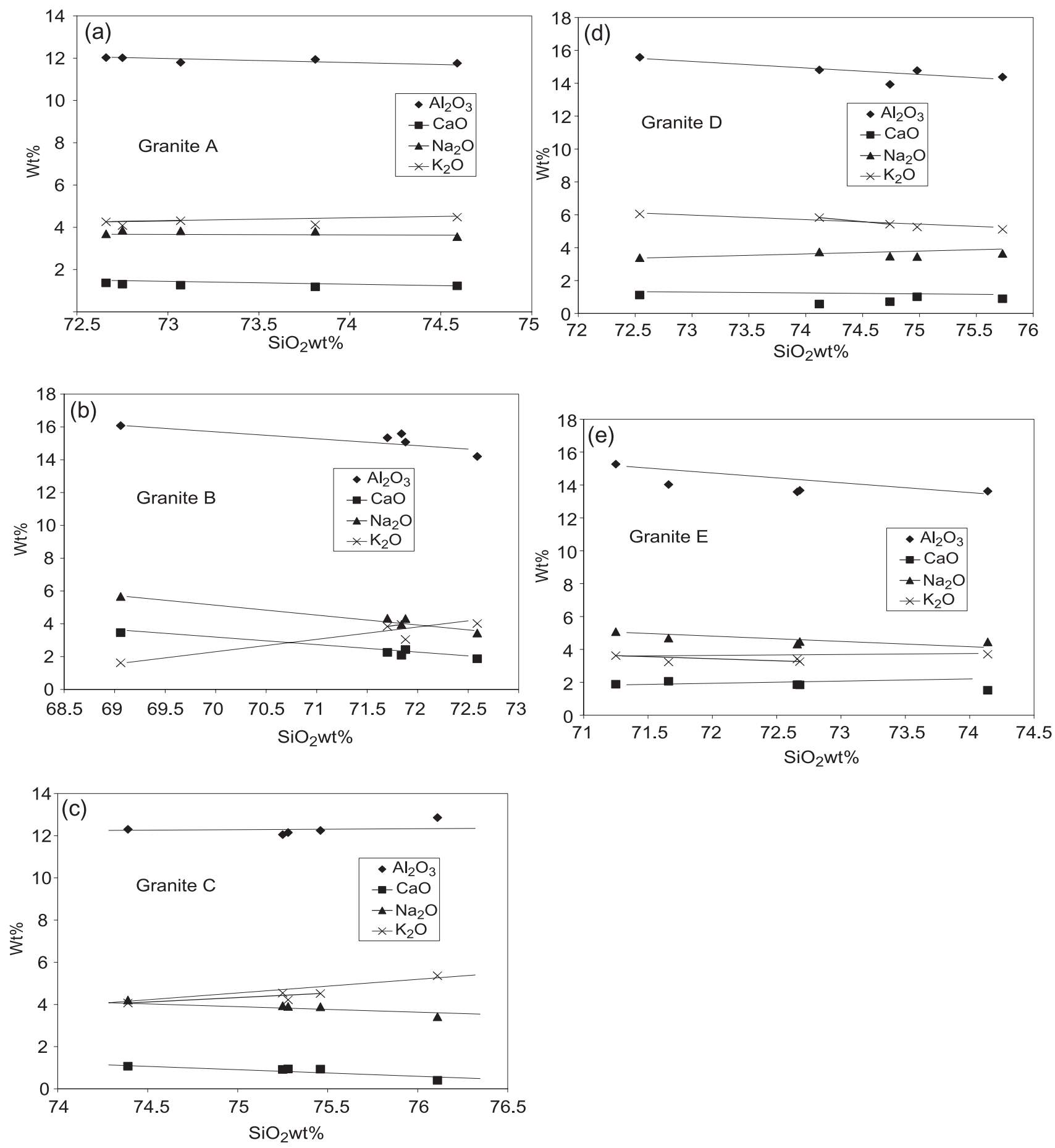

Figure 7. Harker's variation diagrams: $\mathrm{Al}_{2} \mathrm{O}_{3}, \mathrm{CaO}, \mathrm{Na}_{2} \mathrm{O} \& \mathrm{~K}_{2} \mathrm{O}$ against $\mathrm{SiO}_{2}$. A to $\mathrm{E}$ for the respective granitoids.

alumina (A/CNK values between 0.89 and 0.92). A negative correlation between $\mathrm{Si}$ and $\mathrm{Ca}$, and a positive correlation between Si and K, suggest feldspar fractionation to a limited extent (figure 7a).

\subsection{Granite B}

These have high but variable silica contents (between $69 \%$ and $72 \%$ ) and normative compositions between granite and trondhjemite (figure 6).
Also, varying alumina saturation in these rocks is indicated by A/CNK values between 0.92 and 1.06 . Significant feldspar fractionation is indicated by decreasing $\mathrm{Al}, \mathrm{Ca}$ and $\mathrm{Na}$, on the one hand and increasing $\mathrm{K}$ on the other with increasing silica (figure $7 \mathrm{~b}$ ).

\subsection{Granite $C$}

These are high-silica $(>74 \%)$ granites with restricted normative compositions in the granite 
Table 10. Bulk composition of Granite B.

\begin{tabular}{|c|c|c|c|c|c|c|}
\hline $\begin{array}{l}\text { Sample } \\
\text { Ref. no. }\end{array}$ & $\begin{array}{c}\text { SN11 } \\
6\end{array}$ & $\begin{array}{c}\text { SD33 } \\
7\end{array}$ & $\begin{array}{c}\mathrm{S} 11 / 48 \\
8\end{array}$ & $\begin{array}{c}\mathrm{S} 30 / 77 \\
9\end{array}$ & $\begin{array}{c}\mathrm{S} 6 / 30 \\
10\end{array}$ & $\begin{array}{l}\text { S2/8a } \\
\text { Enclave }\end{array}$ \\
\hline $\mathrm{SiO}_{2}$ & 72.79 & 69.06 & 71.88 & 71.7 & 71.84 & 50.82 \\
\hline $\mathrm{TiO}_{2}$ & 0.43 & 0.3 & 0.34 & 0.2 & 0.34 & 1.12 \\
\hline $\mathrm{Al}_{2} \mathrm{O}_{3}$ & 14.2 & 16.08 & 15.08 & 15.34 & 15.59 & 12.35 \\
\hline $\mathrm{Fe}_{2} \mathrm{O}_{3}$ & 2.92 & 2.62 & 2.28 & 1.62 & 1.69 & 14.12 \\
\hline $\mathrm{MnO}$ & 0.02 & 0.07 & 0.02 & 0.02 & 0.02 & 0.18 \\
\hline $\mathrm{MgO}$ & 0.46 & 0.56 & 0.37 & 0.35 & 0.44 & 8.44 \\
\hline $\mathrm{CaO}$ & 1.87 & 3.46 & 2.43 & 2.26 & 2.08 & 10.5 \\
\hline $\mathrm{Na}_{2} \mathrm{O}$ & 3.44 & 5.67 & 4.32 & 4.34 & 3.96 & 2 \\
\hline $\mathrm{K}_{2} \mathrm{O}$ & 4.02 & 1.62 & 3.05 & 3.84 & 3.96 & 0.35 \\
\hline $\mathrm{P}_{2} \mathrm{O}_{5}$ & 0.16 & 0.18 & 0.12 & 0.09 & 0.03 & 0.13 \\
\hline LOI & 0.55 & 0.53 & 0.89 & 0.38 & 0.72 & 0.5 \\
\hline Total & 100.86 & 100.15 & 100.78 & 100.14 & 100.67 & 100.51 \\
\hline $\mathrm{A} / \mathrm{CNK}$ & 1.05 & 0.92 & 1.01 & 1 & 1.06 & \\
\hline $\mathrm{A} / \mathrm{NK}$ & 1.41 & 1.45 & 1.44 & 1.35 & 1.41 & \\
\hline $\mathrm{K} / \mathrm{Na}$ & 1.31 & 0.32 & 0.79 & 0.99 & 1.1 & \\
\hline Mg. no. & 23.61 & 29.51 & 21.67 & 29.75 & 33.78 & 97 \\
\hline \multicolumn{7}{|c|}{ Trace elements in ppm } \\
\hline $\mathrm{Ba}$ & 939 & 449 & 690 & 716 & 934 & 159 \\
\hline $\mathrm{Rb}$ & 78 & 17 & 58 & 55 & 95 & 15 \\
\hline $\mathrm{Sr}$ & 87 & 380 & 203 & 503 & 416 & 131 \\
\hline $\mathrm{Y}$ & 31 & 10 & 6 & 5 & 14 & 24 \\
\hline $\mathrm{Nb}$ & 5 & 3 & 4 & $<$ & 2 & 3 \\
\hline $\mathrm{Zr}$ & 340 & 171 & 179 & 152 & 178 & 55 \\
\hline Th & 72 & $<$ & 23 & 16 & 9 & 2 \\
\hline $\mathrm{U}$ & 3 & $<$ & 1.4 & 1.6 & 5 & 1 \\
\hline $\mathrm{Pb}$ & 31 & 11 & 22 & 37 & 36 & 5 \\
\hline $\mathrm{Ga}$ & 18 & 21 & 18 & 16 & 18 & 18 \\
\hline $\mathrm{Ni}$ & 10 & $<$ & $<$ & $<$ & 3 & 172 \\
\hline $\mathrm{Cu}$ & 6 & 5 & 2 & 7 & 10 & 75 \\
\hline $\mathrm{Zn}$ & 29 & 48 & 20 & 16 & 11 & 113 \\
\hline $\mathrm{Rb} / \mathrm{Sr}$ & 0.9 & 0.05 & 0.29 & 0.11 & 0.23 & 0.11 \\
\hline $\mathrm{Sr} / \mathrm{Y}$ & 2.8 & 38 & 34 & 111 & 30 & 2.38 \\
\hline
\end{tabular}

field (figure 6). However, this granite varies between meta-aluminous and peraluminous compositions (A/CNK values between 0.92 and 1.05). Variation diagrams show decreasing $\mathrm{Ca}, \mathrm{Na} \&$ $\mathrm{Al}$ and increasing $\mathrm{K}$ with increasing silica, which indicate feldspar fractionation in this granite (figure 7c).

\subsection{Granite D}

These are also high-silica $(>74 \%)$ granites of restricted normative composition (figure 6 ) and are peraluminous with $\mathrm{A} / \mathrm{CNK}$ values between 1.08 and 1.16. The strong negative correlation between $\mathrm{Si}$ and $\mathrm{Al}$ as well as between $\mathrm{Si}$ and $\mathrm{K}$, but no correlation between $\mathrm{Si}$ and either $\mathrm{Ca}$ or $\mathrm{Na}$, can only be explained by the variable proportions of garnet and biotite in these rocks (figure $7 \mathrm{~d}$ ).

\subsection{Granite $E$}

These are high-silica $(>71 \%)$ granites, but normative composition varies from granite to trondjhemite (figure 6). These are meta-aluminous with
A/CNK values between 0.93 and 0.97 . Variation of $\mathrm{Al}, \mathrm{Ca}, \mathrm{Na} \& \mathrm{~K}$ in relation to increasing $\mathrm{Si}$, could indicate limited feldspar fractionation, but the slight variation in $\mathrm{CaO}$ (1.51 to $2.06 \%$ ) could be due to variable proportions of other calcic phases, such as epidote and amphibole (figure 7e).

\section{Trace element signatures}

In Granite A, $\mathrm{Rb} / \mathrm{Sr}$ ratios are generally high ( $>1$ ) suggesting biotite in the source rock and there is a negative correlation between $\mathrm{Sr}$ and $\mathrm{Rb}$ (figure 8a) and to some extent between $\mathrm{Sr}$ and $\mathrm{Ba}$; plagioclase in the source rock might have caused such decoupling (Hanson 1978). High Ba and Nb concentrations in these granites (Ba 911 to $1056 \mathrm{ppm}$ and $\mathrm{Nb} 34$ to $38 \mathrm{ppm}$ ) are consistent with their alkali feldspar-dominant modal and bulk chemical compositions. On the other hand, high $\mathrm{Y}$ and $\mathrm{Zr}$ concentrations ( $\mathrm{Y} 71$ to $102 \mathrm{ppm}$ and $\mathrm{Zr} 618$ to $666 \mathrm{ppm}$ ) are presumably due to restitic zircon. High concentrations of $\mathrm{Zn}$ and Ni merely reflect significant modal abundance of opaque oxides. 
Table 11. Bulk composition of Granite $C$.

\begin{tabular}{|c|c|c|c|c|c|}
\hline $\begin{array}{l}\text { Sample } \\
\text { Ref. no. }\end{array}$ & $\begin{array}{c}\mathrm{S} 7 / 37 \\
11\end{array}$ & $\begin{array}{c}\mathrm{S} 7 / 39 \\
12\end{array}$ & $\begin{array}{c}\mathrm{S} 7 / 40 \\
13\end{array}$ & $\begin{array}{c}\text { SD79A } \\
14\end{array}$ & $\begin{array}{c}\text { SD53A } \\
15\end{array}$ \\
\hline $\mathrm{SiO}_{2}$ & 76.11 & 75.25 & 75.28 & 75.46 & 74.39 \\
\hline $\mathrm{TiO}_{2}$ & 0.2 & 0.25 & 0.27 & 0.27 & 0.25 \\
\hline $\mathrm{Al}_{2} \mathrm{O}_{3}$ & 12.86 & 12.05 & 12.15 & 12.25 & 12.3 \\
\hline $\mathrm{Fe}_{2} \mathrm{O}_{3}$ & 1.35 & 3.12 & 3.17 & 3.13 & 3.24 \\
\hline $\mathrm{MnO}$ & 0.05 & 0.08 & 0.07 & 0.07 & 0.08 \\
\hline $\mathrm{MgO}$ & & 0.08 & 0.09 & 0.06 & 0.08 \\
\hline $\mathrm{CaO}$ & 0.4 & 0.92 & 0.94 & 0.93 & 1.07 \\
\hline $\mathrm{Na}_{2} \mathrm{O}$ & 3.42 & 3.94 & 3.91 & 3.89 & 4.21 \\
\hline $\mathrm{K}_{2} \mathrm{O}$ & 5.36 & 4.54 & 4.22 & 4.52 & 4.07 \\
\hline $\mathrm{P}_{2} \mathrm{O}_{5}$ & 0.03 & 0.05 & 0.07 & 0.06 & 0.06 \\
\hline LOI & 0.3 & 0.51 & 0.39 & 0.22 & 0.26 \\
\hline Total & 100.08 & 100.79 & 100.56 & 100.86 & 100.01 \\
\hline $\mathrm{A} / \mathrm{CNK}$ & 1.05 & 0.99 & 0.98 & 1.04 & 1.02 \\
\hline $\mathrm{A} / \mathrm{NK}$ & 1.12 & 1.05 & 1.1 & 1.08 & 1.08 \\
\hline $\mathrm{K} / \mathrm{Na}$ & 1.75 & 1.29 & 1.21 & 1.3 & 1.1 \\
\hline Mg. no. & & 4.77 & 5.26 & 3.6 & 4.61 \\
\hline \multicolumn{6}{|c|}{ Trace elements in ppm } \\
\hline $\mathrm{Ba}$ & 922 & 959 & 887 & 982 & 926 \\
\hline $\mathrm{Rb}$ & 190 & 175 & 167 & 178 & 161 \\
\hline $\mathrm{Sr}$ & 39 & 60 & 54 & 58 & 54 \\
\hline $\mathrm{Y}$ & 101 & 108 & 122 & 109 & 107 \\
\hline $\mathrm{Nb}$ & 28 & 28 & 33 & 25 & 27 \\
\hline $\mathrm{Zr}$ & 392 & 598 & 585 & 590 & 613 \\
\hline Th & 38 & 29 & 23 & 26 & 23 \\
\hline $\mathrm{U}$ & 13 & 10 & 10 & 11 & 9 \\
\hline $\mathrm{Pb}$ & 30 & 37 & 30 & 28 & 29 \\
\hline $\mathrm{Ga}$ & 17 & 18 & 17 & 14 & 15 \\
\hline $\mathrm{Ni}$ & 5 & 19 & 26 & 23 & 20 \\
\hline $\mathrm{Cu}$ & $<$ & 12 & 11 & 11 & 24 \\
\hline $\mathrm{Zn}$ & 47 & 86 & 59 & 61 & 80 \\
\hline $\mathrm{Rb} / \mathrm{Sr}$ & 4.9 & 2.9 & 3.1 & 3.1 & 2.98 \\
\hline $\mathrm{Sr} / \mathrm{Y}$ & 0.39 & 0.56 & 0.44 & 0.53 & 0.5 \\
\hline
\end{tabular}

The multi-element spidergram (figure 9a) shows about 100 times enrichment of the heat-producing elements, K, Rb, Ba, Th \& U, presumably due to the thermal impact of collision.

In Granite $\mathrm{B}$, low $\mathrm{Rb} / \mathrm{Sr}$ ratios could indicate amphibole in the source rock. Negative correlation between $\mathrm{Sr}$ and $\mathrm{Rb}$ (figure 8a) and to some extent between $\mathrm{Sr}$ and $\mathrm{Ba}$ and a positive correlation between $\mathrm{Ca}$ and $\mathrm{Sr}$ (figure 8b) are consistent with plagioclase in the source. Also, a positive correlation between $\mathrm{K}$ and $\mathrm{Ba}$ (figure 8c) possibly reflects plagioclase fractionation. The spidergram (figure $9 \mathrm{~b}$ ) shows enrichment of the incompatible elements, $\mathrm{K}, \mathrm{Rb}, \mathrm{Ba}$, a lack of Sr enrichment, and significant $\mathrm{Y}$ depletion with respect to the mafic granulite enclave. These are explicable by amphibole-dehydration melting, producing a granitic melt and which also involved plagioclase (Rapp and Watson 1995). Also, with respect to the enclave, depletion of $\mathrm{Ti}, \mathrm{Zn}, \mathrm{Ni}$ and $\mathrm{Cu}$ is compatible with the melt-restite relation between the granite and the mafic granulite enclave.
In Granite $\mathrm{C}$, high $\mathrm{Rb} / \mathrm{Sr}$ ratios could indicate biotite in the source. A negative correlation between $\mathrm{Sr}$ and $\mathrm{Rb}$ (figure 8a), but no correlation between $\mathrm{Sr}$ and $\mathrm{Ba}$ could reflect a combination of factors: plagioclase in the source as well as significant feldspar fractionation. The spidergram (figure 9c) shows more than a 100 fold enrichment of $\mathrm{K}, \mathrm{Rb}, \mathrm{Ba}, \mathrm{Th}, \mathrm{U}$ and $\mathrm{Nb}$ with respect to the primitive mantle.

In Granite $\mathrm{D}$, high $\mathrm{Rb} / \mathrm{Sr}$ ratios could indicate biotite in the source. A positive correlation between $\mathrm{Ca}$ and $\mathrm{Sr}$ (figure 8b) and between $\mathrm{Sr}$ and $\mathrm{Ba}$ (figure $8 \mathrm{~d}$ ) could indicate plagioclase in the source. Incidentally, lack of correlation between $\mathrm{Sr}$ and $\mathrm{Rb}$ (figure 8a) and between silica and either $\mathrm{Ca}$ or $\mathrm{Na}$, argue against plagioclase fractionation. As argued in a previous section, variable abundance of peritectic garnet and secondary biotite in these granites could be responsible for these traceelement characteristics. The spidergram (figure 9d) shows significant $\mathrm{Ba}$ enrichment and marginal enrichment in $\mathrm{K}$ and $\mathrm{Rb}$, in the granites relative to the pelitic enclave. The marked Y depletion, but 
Table 12. Bulk composition of Granite D.

\begin{tabular}{|c|c|c|c|c|c|c|}
\hline $\begin{array}{l}\text { Sample } \\
\text { Ref. no. }\end{array}$ & $\begin{array}{c}\mathrm{S} 17 / 89 \\
16\end{array}$ & $\begin{array}{c}\mathrm{S} 18 / 94 \\
17\end{array}$ & $\begin{array}{c}\mathrm{S} 19 / 10 \\
18\end{array}$ & $\begin{array}{c}\mathrm{S} 19 / 6 \\
19\end{array}$ & $\begin{array}{c}\mathrm{S} 20 / 20 \\
20\end{array}$ & $\begin{array}{l}\text { SN34 } \\
\text { Enclave }\end{array}$ \\
\hline $\mathrm{SiO}_{2}$ & 72.54 & 75.73 & 74.98 & 74.12 & 74.74 & 59.94 \\
\hline $\mathrm{TiO}_{2}$ & 0.17 & 0.1 & 0.13 & 0 & 0.01 & 1.2 \\
\hline $\mathrm{Al}_{2} \mathrm{O}_{3}$ & 15.58 & 14.38 & 14.77 & 14.81 & 13.93 & 21.61 \\
\hline $\mathrm{Fe}_{2} \mathrm{O}_{3}$ & 0.98 & 0.65 & 0.74 & 1.17 & 1.54 & 9.39 \\
\hline $\mathrm{MnO}$ & 0.01 & 0.01 & 0.02 & 0.03 & 0.02 & 0.31 \\
\hline $\mathrm{MgO}$ & 0.18 & 0.06 & 0.02 & 0.04 & 0.09 & 1.73 \\
\hline $\mathrm{CaO}$ & 1.12 & 0.89 & 1.01 & 0.57 & 0.71 & 1.14 \\
\hline $\mathrm{Na}_{2} \mathrm{O}$ & 3.39 & 3.65 & 3.46 & 3.74 & 3.48 & 0.96 \\
\hline $\mathrm{K}_{2} \mathrm{O}$ & 6.05 & 5.12 & 5.26 & 5.83 & 5.43 & 3.42 \\
\hline $\mathrm{P}_{2} \mathrm{O}_{5}$ & 0.05 & 0.06 & 0.06 & 0.06 & 0.08 & 0 \\
\hline LOI & 0.61 & 0.51 & 0.7 & 0.38 & 0.8 & 0.48 \\
\hline Total & 100.68 & 101.16 & 101.15 & 100.75 & 100.83 & 100.18 \\
\hline $\mathrm{A} / \mathrm{CNK}$ & 1.1 & 1.16 & 1.11 & 1.09 & 1.08 & 2.94 \\
\hline $\mathrm{A} / \mathrm{NK}$ & 1.28 & 1.24 & 1.29 & 1.18 & 1.2 & 4.09 \\
\hline Mg. no. & 26.49 & 15.25 & 5.03 & 6.28 & 10.27 & 26.53 \\
\hline $\mathrm{K} / \mathrm{Na}$ & 2 & 1.57 & 1.7 & 1.7 & 1.75 & \\
\hline \multicolumn{7}{|c|}{ Trace elements in ppm } \\
\hline $\mathrm{Ba}$ & 1082 & 444 & 1137 & 256 & 328 & 153 \\
\hline $\mathrm{Rb}$ & 184 & 210 & 166 & 175 & 135 & 126 \\
\hline $\mathrm{Sr}$ & 132 & 76 & 124 & 41 & 50 & 67 \\
\hline $\mathrm{Y}$ & 25 & 18 & 29 & 24 & 19 & 51 \\
\hline $\mathrm{Nb}$ & 0 & 1 & $<$ & $<$ & $<$ & 27 \\
\hline $\mathrm{Zr}$ & 329 & 101 & 109 & 15 & 17 & 285 \\
\hline Th & 143 & 23 & 20 & $<$ & $<$ & 17 \\
\hline $\mathrm{U}$ & 12.4 & 14.5 & 11.4 & 12 & 8.4 & 2 \\
\hline $\mathrm{Pb}$ & 63 & 41 & 49 & 46 & 36 & 16 \\
\hline $\mathrm{Ga}$ & 18 & 17 & 19 & 24 & 19 & 33 \\
\hline $\mathrm{Ni}$ & 7 & 1 & 4 & 4 & 2 & 48 \\
\hline $\mathrm{Cu}$ & $<$ & $<$ & 1 & 4 & 2 & 46 \\
\hline $\mathrm{Zn}$ & $<$ & 6 & $<$ & $<$ & 16 & 87 \\
\hline $\mathrm{Rb} / \mathrm{Sr}$ & 1.4 & 2.76 & 1.34 & 4.27 & 2.7 & 1.9 \\
\hline $\mathrm{Sr} / \mathrm{Y}$ & 5.3 & 4.2 & 4.28 & 1.71 & 2.63 & 1.34 \\
\hline
\end{tabular}

little or no $\mathrm{Sr}$ depletion, indicates plagioclase in the source; incidentally, the pelitic enclaves have significant modal abundance of plagioclase. These granites are also depleted in $\mathrm{Ti}, \mathrm{Zn}, \mathrm{Cu}$ and $\mathrm{Ni}$ relative to the pelitic enclave. These complementary trace element signatures are compatible with the restitic nature of the pelitic enclave.

In Granite $\mathrm{E}$, low $\mathrm{Rb} / \mathrm{Sr}$ ratios could indicate amphibole in the source. Lack of correlation between $\mathrm{Sr}$ and $\mathrm{Rb}$ (figure $8 \mathrm{a}$ ) or between $\mathrm{Sr}$ and $\mathrm{Ba}$, along with lack of correlation between $\mathrm{Ca}$ and Sr can only be explained by the formation of magmatic epidote. The spidergram (figure 9e) shows about 100 times enrichment in the heat- producing elements $\mathrm{K}, \mathrm{Rb}, \mathrm{Ba}$, Th and U, relative to the primitive mantle. Also, significant enrichment (about 10 fold) in $\mathrm{Nb}, \mathrm{Sr}$ and $\mathrm{Zr}$ are noted.

It is also important to note that most of these granites have high concentrations of $\mathrm{Zn}$ and $\mathrm{Cu}$, presumably due to the presence of accessory phases as unmelted residual phases, that were not separated from the granitic melt.

\section{Petrogenesis}

Two of the granitoid bodies, namely, $B$ and $D$, could be explained in terms of crustal anatexis, via dehydration melting. The hornblendebearing mafic granulite enclaves (in Granite B) with marginal Mg-enrichment in the hornblendes are consistent with experimental observations of hornblende-dehydration melting (Rapp and Watson 1995). Also, the complementary chemical signatures (figure 9b) between the mafic granulite enclave and the host Granite B, attest to the link between them via hornblende-dehydration melting in the precursors of mafic granulite enclaves. On the other hand, Granite D, with garnetcordierite bearing metapelitic enclaves, inclusions of biotite (more magnesian than that in the matrix) and sillimanite in garnet of the granite, are explicable in terms of biotite-dehydration melting in the precursors of pelitic enclaves. Moreover, more magnesian garnet in the enclave, compared with those of the common metapelite assemblage, 
Table 13. Bulk composition of Granite E.

\begin{tabular}{|c|c|c|c|c|c|}
\hline $\begin{array}{l}\text { Sample } \\
\text { Ref. no. }\end{array}$ & $\begin{array}{c}\mathrm{S} 13 / 61 \\
21\end{array}$ & $\begin{array}{c}\mathrm{S} 13 / 62 \\
22\end{array}$ & $\begin{array}{c}\mathrm{S} 13 / 63 \\
23\end{array}$ & $\begin{array}{c}\mathrm{S} 16 / 86 \\
24\end{array}$ & $\begin{array}{c}\mathrm{SD} 34 \mathrm{~B} \\
25\end{array}$ \\
\hline$\overline{\mathrm{SiO}_{2}}$ & 72.66 & 74.14 & 71.66 & 71.25 & 72.68 \\
\hline $\mathrm{TiO}_{2}$ & 0.29 & 0.17 & 0.32 & 0.2 & 0.25 \\
\hline $\mathrm{Al}_{2} \mathrm{O}_{3}$ & 13.58 & 13.62 & 14.03 & 15.27 & 13.67 \\
\hline $\mathrm{Fe}_{2} \mathrm{O}_{3}$ & 2.77 & 1.88 & 3.17 & 2.11 & 2.67 \\
\hline $\mathrm{MnO}$ & 0.04 & 0.03 & 0.05 & 0.04 & 0.04 \\
\hline $\mathrm{MgO}$ & 0.47 & 0.28 & 0.46 & 0.42 & 0.5 \\
\hline $\mathrm{CaO}$ & 1.85 & 1.51 & 2.06 & 1.88 & 1.84 \\
\hline $\mathrm{Na}_{2} \mathrm{O}$ & 4.32 & 4.46 & 4.69 & 5.08 & 4.48 \\
\hline $\mathrm{K}_{2} \mathrm{O}$ & 3.42 & 3.71 & 3.24 & 3.62 & 3.26 \\
\hline $\mathrm{P}_{2} \mathrm{O}_{5}$ & 0.12 & 0.1 & 0.13 & 0.07 & 0.12 \\
\hline LOI & 0.76 & 0.75 & 0.95 & 0.89 & 0.75 \\
\hline Total & 99.49 & 99.9 & 99.81 & 99.89 & 99.51 \\
\hline $\mathrm{A} / \mathrm{CNK}$ & 0.95 & 0.96 & 0.93 & 0.94 & 0.93 \\
\hline $\mathrm{A} / \mathrm{NK}$ & 1.21 & 1.2 & 1.25 & 1.24 & 1.25 \\
\hline Mg. no. & 24.95 & 22.59 & 22.13 & 28.16 & 26.86 \\
\hline $\mathrm{K} / \mathrm{Na}$ & 0.89 & 0.93 & 0.77 & 0.8 & 0.81 \\
\hline \multicolumn{6}{|c|}{ Trace elements in ppm } \\
\hline $\mathrm{Ba}$ & 625 & 714 & 635 & 541 & 586 \\
\hline $\mathrm{Rb}$ & 95 & 95 & 130 & 112 & 88 \\
\hline $\mathrm{Sr}$ & 225 & 221 & 255 & 267 & 238 \\
\hline $\mathrm{Y}$ & 29 & 18 & 32 & 18 & 27 \\
\hline $\mathrm{Nb}$ & 13 & 7 & 13 & 10 & 13 \\
\hline $\mathrm{Zr}$ & 258 & 161 & 280 & 180 & 252 \\
\hline Th & 22 & 11 & 11 & 15 & 13 \\
\hline $\mathrm{U}$ & 4.2 & 4.7 & 6.7 & 5.8 & 3.7 \\
\hline $\mathrm{Pb}$ & 31 & 22 & 31 & 36 & 27 \\
\hline $\mathrm{Ga}$ & 21 & 18 & 19 & 19 & 20 \\
\hline $\mathrm{Ni}$ & 13 & 4 & 12 & 4 & 6 \\
\hline $\mathrm{Cu}$ & 17 & 16 & 13 & 16 & 15 \\
\hline $\mathrm{Zn}$ & 41 & 21 & 44 & 28 & 38 \\
\hline $\mathrm{Rb} / \mathrm{Sr}$ & 0.42 & 0.43 & 0.51 & 0.42 & 0.37 \\
\hline $\mathrm{Sr} / \mathrm{Y}$ & 7.75 & 12.27 & 7.97 & 14.83 & 8.81 \\
\hline
\end{tabular}

attest to the restitic nature of this enclave (Le Breton and Thompson 1988; Patino Douce and Johnston 1991). Complementary chemical signatures (figure 9d) between the pelitic enclave and the host Granite D, attest to the link between them via biotite-dehydration melting in pelitic precursors.

Although, the other granitoid bodies can not be so linked with the enclaves, in terms of dehydration melting, the presence of enclaves from both the high-grade and low-grade terrains, would imply that precursors of these granitoids included lithologies of the two adjacent belts.

\section{Relation to tectonism}

Bhattacharya (1997) has described an oblique collisional (transpression) juxtaposition of the Eastern Ghats belt against the stable craton of Singhbhum to the north. It is noteworthy that all the lithologies in the boundary area, including the granitoids, have the imprints of a shear cleavage, presumably developed at the time of collision. In the granitoids, this shear cleavage is often defined by an anastomosing fabric around feldspar porphyroclasts (figure 2c). Other interesting microscopic fabrics are: marginal granulation and sub-grain formation in K-feldspars (figure 2f) and replacement of K-feldspar by myrmekite (figure $2 \mathrm{~g}$ ). Also the common evidence of high-temperature solid-state deformation in the granitoids would suggest their syn-tectonic emplacement during collision (figures $3 \mathrm{a}-\mathrm{c}$ ). Together with the field features, these microscopic features attest to the syn-tectonic emplacement of the granitoids. The alternative of pre-tectonic granite seems unlikely in view of the fact that the precursor lithologies (represented by the enclaves) belong to the two different terrains, which were juxtaposed only during collision. Also, the high-temperature solidstate deformation fabrics would, in that case, have been associated with later heating and metamorphic effects, which is characteristically absent. On the contrary, retrograde reaction textures, such as biotite after garnet (figure 2d) are commonly observed. Thus the high- to relatively high-temperature solid-state deformation 

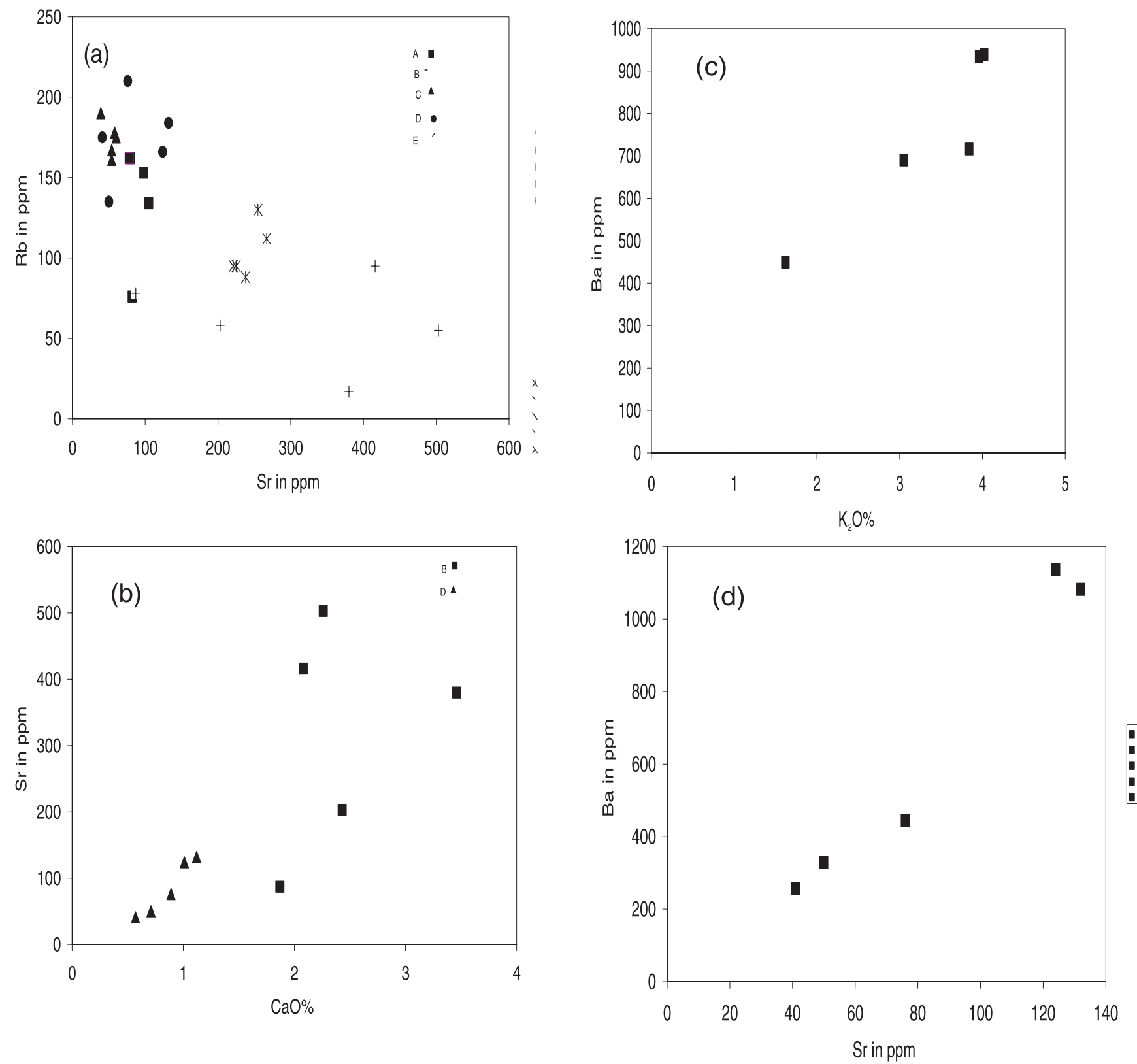

Figure 8. Trace element correlation diagrams, A to E. Symbols: Square $=$ A, Plus $=$ B, Triangle $=$ C, Circle $=$ D, Asterix $=$ E.

fabrics in the granitoids is thought to have developed during cooling from magmatic temperatures (Vernon et al 1983).

\section{Conclusions}

The craton-mobile belt relationship could be a key factor in Precambrian crustal evolution across a terrain boundary. Marked by a crustal-scale shear zone, the western margin of EGMB against Bastar craton implies rapid exhumation of deep-crustal granulites during collision (Bhattacharya 2004). The northern margin of EGMB against the Singhb- hum craton, on the other hand, is marked by granitic magmatism, closely following the granulite facies event, and may imply decompression melting and exhumation of deep crustal rocks at the same time.

\section{Acknowledgements}

This research was sponsored by the Department of Science \& Technology, Govt. of India, under a Project ESS/CA/A-9/15/92. Indian Statistical Institute provided the infrastructural facilities. Analytical facilities provided by the Wadia 

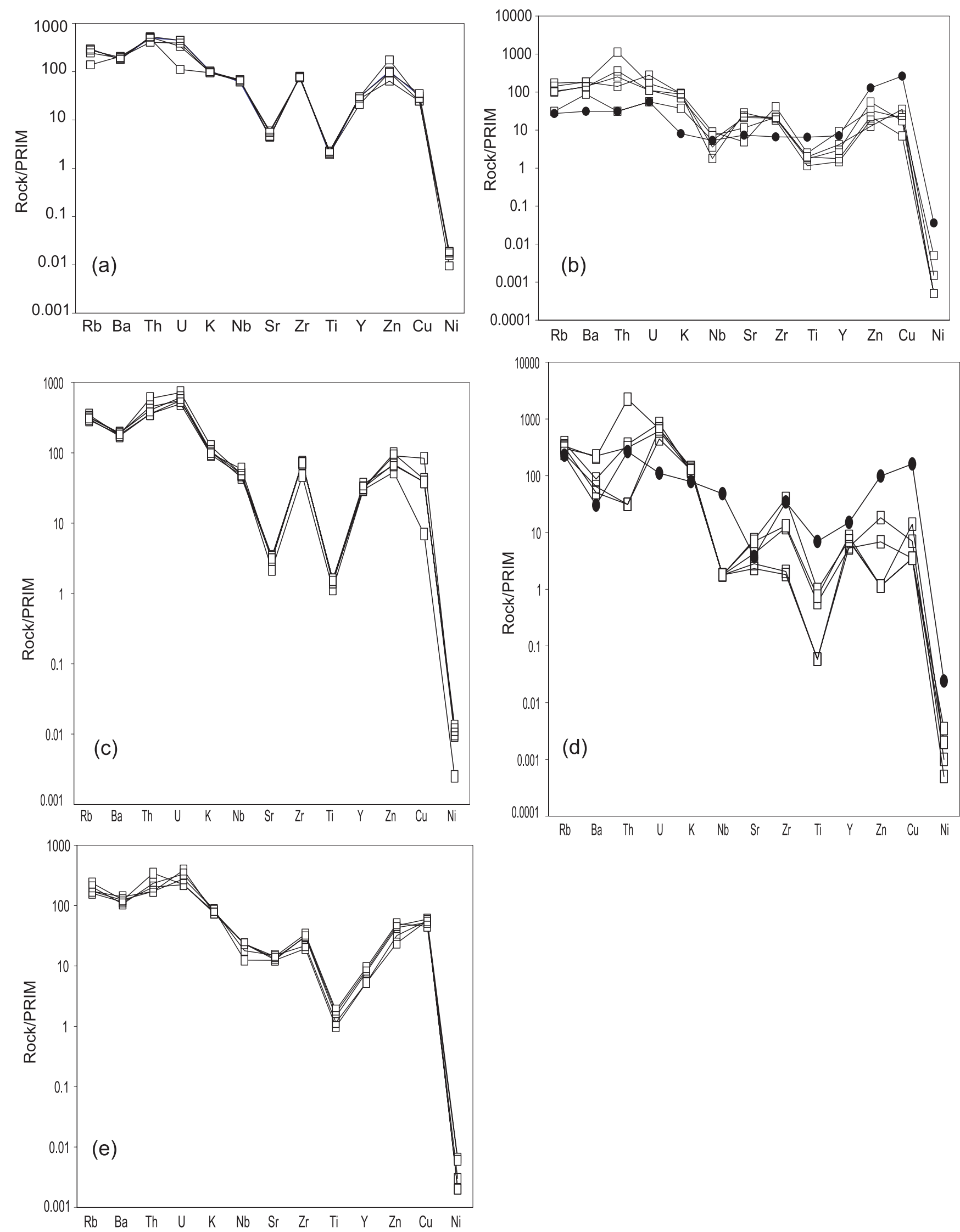

Figure 9. Multi-element spider plot of the granitoids. A to E for the respective granitoids. For B and D granitoids, the restitic enclaves are also plotted. Symbols: open square for granitoids and solid circle for the enclaves. 
Institute of Himalayan Geology and Roorkee University are thankfully acknowledged. We thankfully acknowledge critical comments from S C Patel, Thomas Frisch and an anonymous reviewer.

\section{References}

Banerjee P K, Mahakud S P, Bhattacharya A K and Mohanty A K 1987 On the northern margin of the Eastern Ghats in Orissa; Record, G.S.I. 118 1-8

Berman R G 1988 Internally consistent thermodynamic data for minerals in the system $\mathrm{Na}_{2} \mathrm{O}-\mathrm{K}_{2} \mathrm{O}-\mathrm{CaO}-\mathrm{MgO}-$ $\mathrm{FeO}-\mathrm{Fe}_{2} \mathrm{O}_{3}-\mathrm{Al}_{2} \mathrm{O}_{3}-\mathrm{SiO}_{2}-\mathrm{TiO}_{2}-\mathrm{H}_{2} \mathrm{O}-\mathrm{CO}_{2}$, J. Petrology 29 $445-522$

Berman R G 1990 Mixing properties of Ca-Mg-Fe-Mn garnets; Amer. Mineralogists 75 328-344

Berman R G 1991 Thermobarometry using multiequilibrium calculations: a new technique, with petrological applications; Can. Mineralogist 29 833-855

Bhattacharya S 1993 Refinement of geothermometry for cordierite granulites; Proc. Indian Acad. Sci. (Earth Planet Sci.) 102 537-545

Bhattacharya S 1997 Evolution of Eastern Ghats granulite belt of India in a compressional tectonic regime and juxtaposition against Iron Ore craton of Singhbhum by oblique collision-transpression; Proc. Indian Acad. Sci. (Earth Planet. Sci.) 106 65-75

Bhattacharya S 2004 High-temperature crustal-scale shear zone at the western margin of the Eastern Ghats belt; implications for rapid exhumation; J. Asian Earth Sci. (in press)

Bhattacharya S, Kar Rajib, Misra S and Teixeira W 2001 Early Archaean continental crust in the Eastern Ghats granulite belt, India: Isotopic evidence from a charnockite suite; Geol. Mag. 138 609-618

Dasgupta S, Sengupta P, Mondal A and Fukuoka M 1993 Mineral chemistry and reaction textures in metabasites from the Eastern Ghats belt, India and their implications; Mineralogical Magazine 57 113-120

Hanson G N 1978 The application of trace elements to the petrogenesis of igneous rocks of granitic composition; Earth. Planet. Sci. Lett. 38 26-43

Kar Rajib, Bhattacharya S and Sheraton J W 2003 Hornblende-dehydration melting in mafic rocks and the link between massif-type charnockite and associated granulites, Eastern Ghats granulite belt, India; Contrib. Mineral. Petrol. 145 707-729

Le Breton N and Thompson A B 1988 Fluid-absent (dehydration) melting of biotite in metapelites in the early stages of crustal anatexis; Contrib. Mineral. Petrol. 99 $226-237$
Mahalik N K 1994 Geology of the contact between the Eastern Ghats belt and North Orissa craton, India; J. Geol. Soc. India 44 41-51

Misra S, Moitra S, Bhattacharya S and Sivaraman T V 2000 Archaean granitoids at the contact of Eastern Ghats granulite belt and Singhbhum craton, in Bhuban-Rengali sector, Orissa, India; Gondwana Research 3 205-213

Moitra S 1996 Boundary relations (structural and metamorphic) between Eastern Ghats and Singhbhum, in the area around Bhuban, Orissa; Indian J. Earth Sci. 23 $1-12$

Mukhopadhaya A K and Bhattacharya A 1997 Tectonothermal evolution of the gneiss complex at Salur in the Eastern Ghats granulite belt of India; J. Met. Geol. 15 719-734

O'Connor J T 1965 A classification for quartz-rich igneous rocks based on feldspar ratios; US Geological Survey Professional paper 525B 79-84

Paterson S R, Vernon R H and Tobisch O T 1989 A review of criteria for the identification of magmatic and tectonic foliations in granitoids; J. Struc. Geol. 11 349-363

Patino Douce A E and Johnston A D 1991 Phase equilibrium and melt productivity in the pelitic system: implications for the origin of peraluminous granitoids and aluminous granulites; Contrib. Mineral. Petrol. 107 2202-2218

Patra P C, Panda P K and Mitra S N 1994 The contact between the Eastern Ghats granulite belt and the Iron Ore Super Group, some observations around Rengalbeda - Deogarh Dist, Orissa (Abstract), In : Workshop on Eastern Ghats Mobile Belt (India: Vishakapatnam) $18-19$

Rapp R P and Watson E B 1995 Dehydration melting of metabasalt at 8-32 kbar: Implications for continental growth and crust-mantle recycling; J. Petrology 36 891-931

Sengupta P, Dasgupta S, Bhattacharya P K, Fukuoka M, Chakraborti S and Bhowmik S 1990 Petrotectonic imprints in the sapphirine granulites from Anantagiri, Eastern Ghats mobile belt, India; J. Petrology 31 971-996

Simpson C and Wintsch R P 1989 Evidence for deformationinduced K-feldspar replacement by myrmekite; J. Met. Geol. 7 261-275

Streckeisen A L 1976 To each plutonic rock its proper name; Earth Science Review 12 1-33

Tulloch A J 1986 Comments on "implications of magmatic epidote bearing plutons on crustal evolution in the accreted terranes of north western North America" and "Magmatic epidote and its petrologic significance"; Geology 14 186-187

Vernon R H, Williams V A and D'Arcy W F 1983 Grainsize reduction and foliation development in a deformed granitoid batholith; Tectonophysics 92 123-145 